\title{
Assessment of PIV-based analysis of water entry problems through synthetic numerical datasets
}

\author{
Andrea L. Facci ${ }^{\mathrm{a}, *}$, Riccardo Panciroli ${ }^{\mathrm{b}}$, Stefano Ubertini ${ }^{\mathrm{a}}$, Maurizio Porfiri ${ }^{\mathrm{c}}$ \\ ${ }^{a}$ School of Engineering, University of "Tuscia", 01100 Viterbo, Italia. \\ ${ }^{b}$ Università degli studi Niccolò Cusano, 00166 Roma, Italia. \\ ${ }^{c}$ Department of Mechanical and Aerospace Engineering, New York University Polytechnic School of \\ Engineering, Brooklyn, NY, 11201 USA.
}

\begin{abstract}
The phenomenon of hull-slamming, that is, the sudden impact of a solid body on the water surface, is critical in the design of naval structures. Thus, the development and validation of schemes to predict the slamming load and elucidate energy exchange during water entry are of fundamental importance in a wide range of engineering applications. Recent studies have demonstrated the possibility of using direct flow measurements from particle image velocimetry (PIV) to investigate the kinetics of water entry. Specifically, these efforts have contributed a first characterization of the hydrodynamic loading on impacting wedges and of the energy imparted to the water pile-up and the spray jets. Here, we seek to provide a thorough assessment of such a PIV-based approach through synthetic datasets, in which PIV parameters, such as the camera acquisition rate and the size of the interrogation area, are systematically varied, without experimental confounds. We implement a direct computational framework to study the two-dimensional flow physics generated during the water entry of a rigid wedge. Water and air are treated as immiscible phases and their relative motion is utilized to track the free surface dynamics. Our results show that the PIV-based methodology allows for an accurate reconstruction of the pressure field from the measured velocity field, except for early stages of the impact and for a small region close to the free surface. We also demonstrate that the reconstruction is only marginally affected by the spatial resolution, while a sufficiently high acquisition frequency is required to correctly predict the pressure field in the pile-up region. The proposed computational framework can also find application in the analysis of untapped aspects of water entry problems, such as cycling loading, flow transitions and separation, and the spray jets.
\end{abstract}

Keywords: Computational fluid dynamics, hull slamming, particle image velocimetry, pressure reconstruction, water entry

\footnotetext{
${ }^{*}$ Corresponding author. Tel.: +390761 357676 .

Email addresses: andrea.facci@unitus.it (Andrea L. Facci), riccardo.panciroli@unicusano. it (Riccardo Panciroli), stefano.ubertini@unitus.it (Stefano Ubertini), mporfiri@nyu.edu (Maurizio Porfiri)
}

Preprint submitted to Journal of Fluids and Structures

April 2, 2015 


\section{Introduction}

Predicting the hydrodynamic loading on a structure impacting the water surface is of critical importance in the design of naval and aeronautical structures (Hughes et al., 2013). The seminal work by Von Karman (1929) resulted in the first analytical model of the vertical water entry of a rigid body. This model has been later extended to account for the pile-up phenomenon by Wagner (1932). Several analytical models have been developed since then (Dobrovol'skaya, 1969; Korobkin, 2006; Tassin et al., 2010; Khabakhpasheva and Korobkin, 2013; Tassin et al., 2013; Qin and Batra, 2009). Despite the capabilities of some of these analytical schemes to deal with complex geometries (Korobkin, 2006) and flexible bodies (Tassin et al., 2013; Qin and Batra, 2009), numerical models are foreseen to be preferred (Hirdaris and Temarel, 2009; Kapsenberg, 2011; Hughes et al., 2013) for their accuracy and versatility. Thus, many numerical models have been proposed for the analysis of water entry problems, ranging from traditional computational fluid dynamics (CFD) solvers (Colicchio et al., 2006; Sun and Faltinsen, 2006; Das and Batra, 2011) to meshless methods (Oger et al., 2006; Shao, 2009; Gong et al., 2009; Panciroli et al., 2012) and Lattice Boltzmann solvers (De Rosis et al., 2014; Zarghami et al., 2014). The main challenges in the numerical analysis of water entry problems are associated with the impulsive nature of the impact, which may lead to large numerical oscillations (Hirsch, 2007), and the existence of a free surface, whose motion must be correctly tracked by the numerical solver. A close prediction of the water free surface evolution is particularly relevant for the estimation of the slamming load experienced by the body (Abrate, 2013).

While analytical and computational models enable a thorough and systematic analysis of both the kinematics and kinetics of the water flow and their relationship to the spatiotemporal evolution of the slamming load, experimental studies have primarily focused on the wedge motion and the total vertical force on the wedge during impact (Peterson et al., 1997; Faltinsen, 2000; Carcaterra and Ciappi, 2004; Judge et al., 2004; Wu et al., 2004; Tveitnes et al., 2008; Luo et al., 2012; Panciroli et al., 2013). Only few efforts have documented a direct measurement of the hydrodynamic loading at selected locations on the impacting structure through pressure gauges (Charca et al., 2009; Battley and Allen, 2011; Stenius et al., 2013). Recently, a non-invasive contactless experimental methodology based on particle image velocimetry (PIV) (Raffel et al., 1998; Van Oudheusden, 2013) has been proposed to experimentally reconstruct the pressure field in water entry problems (Panciroli and Porfiri, 2013; Nila et al., 2013; Panciroli et al., 2015; Shams et al., accepted for publication; Jalalisendi et al., accepted for publication). This methodology enables the estimation of the slamming load on the whole structure that is wetted by the water and of the energy imparted to the pile-up and the spray jets during impact. Such a methodology can thus potentially bridge the gap between experiments and detailed numerical and analytical studies.

The PIV-based methodology reconstructs the pressure field through the integration of Navier-Stokes (Panciroli and Porfiri, 2013; Panciroli et al., 2015; Jalalisendi et al., accepted for publication) or Poisson's (Nila et al., 2013; Shams et al., accepted for publication) equations using the velocity data from PIV measurements. Results presented in (Panciroli and Porfiri, 2013; Panciroli et al., 2015; Nila et al., 2013; Shams et al., accepted for publication; 
Jalalisendi et al., accepted for publication) demonstrate the possibility of estimating the distributed hydrodynamic loading on wedges of different deadrise angle and curvature as well as dissecting the energy imparted to the pile-up and the spray jets. However, the effect of PIV parameters, such as acquisition rate and size of the interrogation region, on the accuracy of the approach are presently untested. Here, we seek to establish a thorough assessment of PIV-based analysis of water entry through simulation data, in which PIV parameters can be varied in a broad range, even beyond current imaging constraints, without experimental confounds. We numerically simulate the water entry of a rigid wedge under free fall, in the same experimental conditions of Panciroli and Porfiri (2013).

While all our previous work on PIV-based pressure reconstruction is based on experimental data (Panciroli and Porfiri, 2013; Panciroli et al., 2015; Shams et al., accepted for publication; Jalalisendi et al., accepted for publication), this work is focused on synthetic data. Specifically, the main contribution of this work is the development of a CFD toolbox for the analysis of water entry problem. Such a toolbox enables a thorough assessment of the PIV-based pressure reconstruction methodology (Panciroli and Porfiri, 2013; Nila et al., 2013; Panciroli et al., 2015; Shams et al., accepted for publication; Jalalisendi et al., accepted for publication), without confounds associated with experimental uncertainties and practical limitations of current PIV technology. Through this synergic dataset, we can systematically dissect the role of several parameters for PIV analysis, including the image resolution and the acquisition rate, and ascertain the accuracy of our pressure reconstruction algorithm to cope with boundary conditions at the moving wedge and the moving surface. Beyond the analysis undertaken in this work, which is focused on the algorithm proposed in (Panciroli and Porfiri, 2013; Panciroli et al., 2015), this synthetic dataset can be used to verify PIV based pressure reconstruction based on Poisson's equation (Nila et al., 2013; Shams et al., accepted for publication).

The paper is organized as follows. In Section 2, we provide a concise statement of the problem addressed in this study. Therein, we also define the main geometric and physical parameters. In Section 3, we briefly describe the experimental setup along with the methodology used to reconstruct the pressure field and the fluid energy from PIV measurements. In Section 4, we describe the numerical scheme to simulate the free surface fluid flow. In Section 5, we compare numerical and experimental findings, in terms of hydrodynamic load, pressure and velocity fields, pile-up and mechanical energy transferred to the flow. Therein, we also verify the proposed pressure reconstruction methodology through a refined numerical dataset and we study the sensitivity of the methodology with respect to the resolution of the interrogation area and the camera acquisition frequency. Conclusions are summarized in Section 6. In AppendixA, we assess the grid convergence and validate the numerical method through comparison with independent results in the literature.

\section{Problem statement}

We study the hydrodynamics associated with the vertical water impact of a rigid wedge. The problem is sketched in Fig. 1, along with the main geometric parameters. A Cartesian frame of reference is placed with the $x$-axis along the water free surface, prior to impact, 


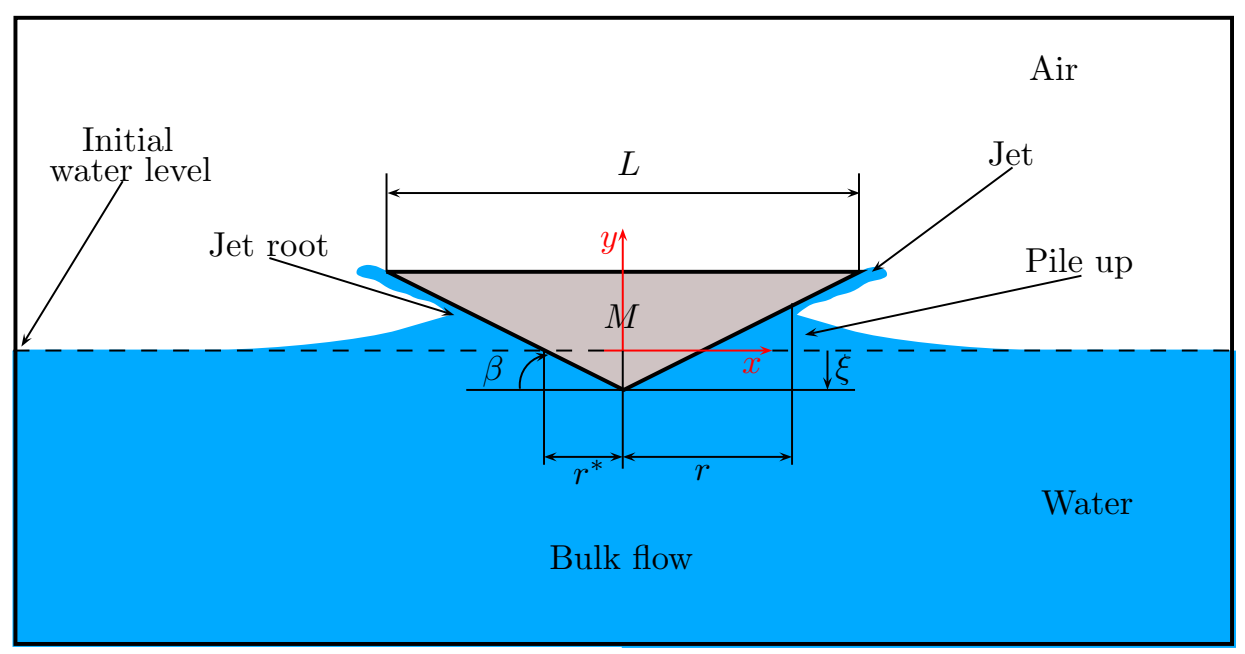

Figure 1: Sketch of the problem in study. The bulk flow represents the water region below the initial water level.

and $y$-axis along the wedge symmetry axis. We denote with $\beta$ the wedge deadrise angle and with $L$ its span, which is assumed to be much smaller than its width (along the axis orthogonal to the $x y$-plane), so that the problem can be treated as two-dimensional. We refer to $\xi(t)$ as the entry depth of the keel, at time $t$, and we set $\xi(0)=0$. Moreover, we identify with $r^{*}=\xi / \tan \beta$ the reference wetted length. Due to the pile-up phenomenon the effective wetted length $r$ is larger than $r^{*}$, and we define the pile-up coefficient as $\phi=r / r^{*}$ (Abrate, 2013). The bulk flow (the water region below the undisturbed free surface) and the pile-up and the spray jets are illustrated in Fig. 1.

We focus on impact speeds much smaller that the speed of sound, so that the flow can be treated as incompressible (De Rosis et al., 2014). Air and water are assumed to be immiscible, so that the flow field is described by the following equations for the conservation of mass and momentum:

$$
\begin{aligned}
\nabla \cdot \boldsymbol{u} & =0, \\
\frac{\mathrm{D}(\rho \boldsymbol{u})}{\mathrm{D} t} & =-\nabla p+\nabla \cdot \mu \nabla \boldsymbol{u}+\rho \boldsymbol{g},
\end{aligned}
$$

where $\boldsymbol{u}$ is the fluid (air or water) velocity, $p$ is the pressure, $\mu$ is the dynamic viscosity, $\boldsymbol{g}$ is the gravitational acceleration, and $\mathrm{D}(\cdot) / \mathrm{D} t$ stands for material derivative. In addition, $\rho$ is the fluid mass density, which equals the constant values $\rho_{\mathrm{a}}$ or $\rho_{\mathrm{w}}$ for air or water, respectively. The force exerted by the fluid, air and water, on the wedge is

$$
\boldsymbol{F}=\int_{\Sigma} \boldsymbol{T} \boldsymbol{n} \mathrm{d} \Sigma,
$$

where

$$
\boldsymbol{T}=-p \boldsymbol{I}+\mu\left[\nabla \boldsymbol{u}+(\nabla \boldsymbol{u})^{\mathrm{T}}\right]
$$


is the Cauchy stress tensor, $\Sigma$ is the wedge surface, and $\boldsymbol{n}$ is the unit normal to the wedge. The motion of the wedge against the fluid increases the mechanical energy of the fluid of a quantity equal to the work $W_{\text {wedge }}$ done by the wedge

$$
W_{\text {wedge }}=\int_{0}^{\xi} F_{y} \mathrm{~d} \xi \text {. }
$$

where $F_{y}$ is the vertical component of $\boldsymbol{F}$. In turn, the mechanical energy in the whole fluid domain $\Omega$ is

$$
E=\int_{\Omega}\left(\frac{1}{2} \rho|\boldsymbol{u}|^{2}+\rho g y\right) \mathrm{d} \Omega,
$$

where $g$ is the magnitude of $\boldsymbol{g}$. Thus, $E$ accounts for both the kinetic and gravitational potential energy in air and water. We refer to the energy fraction associated with the bulk flow as $E_{\text {bulk }}$, whereby the integral in Eq. (5) is restricted to the water region below the undisturbed free surface. For free fall experiments (Panciroli et al., 2015), the fluid energy can can also be related to the energy of the wedge

$$
E_{\text {wedge }}=\frac{1}{2} M \dot{\xi}^{2}-M g \xi
$$

where $M$ is the wedge dry mass per unit depth and a superimposed dot denotes time differentiation.

\section{Experimental analysis of water impact}

Our study is based on PIV (Raffel et al., 1998; Van Oudheusden, 2013). Here, we briefly summarize the experimental setup and the procedure utilized to evaluate the pressure field in the water and estimate the fluid energy; further details can be found in (Panciroli and Porfiri, 2013; Panciroli et al., 2015).

\subsection{Experimental setup}

The experimental apparatus consists of a water tank $800 \mathrm{~mm}$ large, $320 \mathrm{~mm}$ wide, and $350 \mathrm{~mm}$ deep, that is suspended at $800 \mathrm{~mm}$ from the floor. Two prismatic rails are fixed on top of the tank to constrain the free fall of the wedge. A green laser is located below the tank to illuminate a thin sheet of water at the mid-span of the falling wedge, where the fluid flow can be considered two-dimensional due to symmetry. A high-speed camera is placed in front of the water tank to record the water flow at a frequency rate of $4 \mathrm{kHz}$. Water is seeded with silver-coated hollow glass spheres (Melling, 1997; Murakami and Ichikawa, 1989) with mean diameter of $44 \mu \mathrm{m}$ to aid PIV analysis, and the velocity field is obtained from the particles' displacement, obtained from image correlation (Raffel et al., 1998; Van Oudheusden, 2013).

The PIV analysis is performed using the open source code PIVlab developed by Thielicke and Stamhuis (2014a) and Thielicke and Stamhuis (2014b). 


\subsection{Pressure reconstruction}

PIV yields a uniform grid of velocity vectors distributed over the portion of the image occupied by the seeded water. Our pressure reconstruction relies on the numerical integration of Eq. (1b), where we neglect viscosity (Panciroli and Porfiri, 2013) and we focus on the water phase, for which velocity data are available. Thus, Eq. (1b) is specialized to

$$
\nabla p=\rho_{\mathrm{w}}\left(-\frac{\partial \boldsymbol{u}}{\partial t}-\boldsymbol{u} \cdot \nabla \boldsymbol{u}+\boldsymbol{g}\right)
$$

Velocity field extracted from PIV are smoothed through a multidimensional spline smoothing technique (Garcia, 2010). The pressure gradient is first integrated along the horizontal and vertical boundaries of the PIV field of view, using forward integration (Panciroli and Porfiri, 2013). Then, the pressure field is reconstructed through the spatial eroding scheme by Baur and Köngeter (1999). Through image masking, the approach accounts for the motion of both the wedge and the free surface.

Central to the analysis are two parameters: the size of the PIV interrogation area and the acquisition frequency. The interrogation area is an $n \times n$ pixels window over which image cross-correlation is executed by PIVlab. The accuracy of the results and the spatial resolution of the velocity grid are generally affected by this parameter (Willert and Gharib, 1991; Thielicke and Stamhuis, 2014a). The acquisition frequency instead relates to the maximum velocity that can be estimated (Willert and Gharib, 1991; Thielicke and Stamhuis, 2014a). While a larger acquisition frequency is desirable to resolve large flow speeds, increasing the acquisition rate generally reduces the size of the image (due to limitations on the data rate that can be elaborated by the camera).

\subsection{Estimation of the energy transferred to the flow bulk during impact}

PIV can be further used to accurately estimate the energy imparted to the flow bulk as elaborated in (Panciroli et al., 2015). Specifically, the energy in the half of the fluid bulk is estimated as

$$
E_{\mathrm{bulk}}(t)=\int_{\widetilde{\Omega}_{\mathrm{bulk}}}\left(\frac{1}{2} \rho_{\mathrm{w}}|\boldsymbol{u}|^{2}+\rho_{\mathrm{w}} g y\right) \mathrm{d} \widetilde{\Omega}+\int_{0}^{t}\left[\int_{\Gamma_{1} \cup \Gamma_{2}}\left(\frac{1}{2}|\boldsymbol{u}|^{2}+g y+\frac{p}{\rho_{\mathrm{w}}}\right) \rho_{\mathrm{w}}(\boldsymbol{u} \cdot \boldsymbol{n}) \mathrm{d} \Gamma\right] \mathrm{d} \tau
$$

where $\widetilde{\Omega}_{\text {bulk }}$ is the part of the fluid bulk captured by PIV and $\Gamma_{1}$ and $\Gamma_{2}$ are the portions of the perimeter of the PIV domain sketched Fig. 2. Thus, the first term on the right hand side of Eq. (8) is the energy in the portion of the fluid bulk captured by the PIV field of view. The second term on the right hand side of Eq. (8) is the amount of energy that leaves $\widetilde{\Omega}_{\text {bulk }}$ through $\Gamma_{1}$ and $\Gamma_{2}$ towards the rest of the fluid bulk.

The velocity field in $\widetilde{\Omega}_{\text {bulk }}$ is directly evaluated from PIV data and the pressure $p$ on $\Gamma_{1}$ and $\Gamma_{2}$ is estimated using the reconstruction technique above. The time integration is performed using a forward difference scheme and assuming that the fluid is initially at rest. 


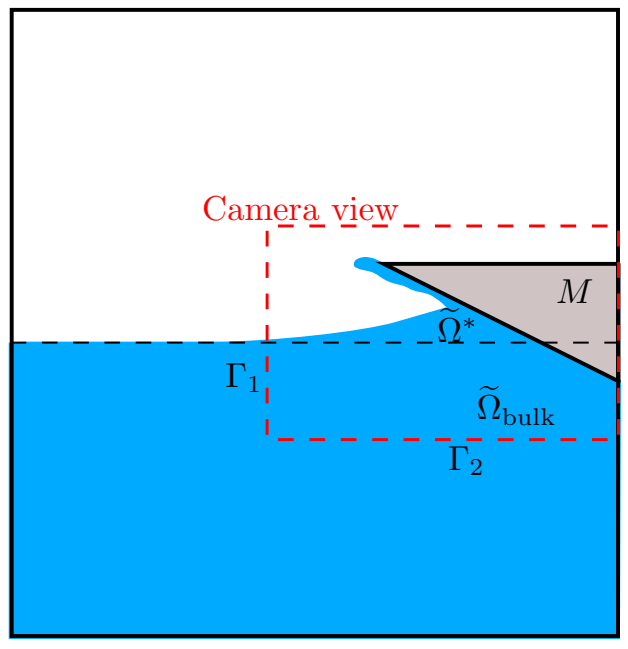

Figure 2: Schematics of the control volume analysis with overlaid notations.

From the energy in the fluid bulk in Eq. (8) and the knowledge of the wedge motion, we estimate the energy imparted to the risen water (pile-up and the spray jet) identified as $\widetilde{\Omega}^{*}$ in Fig. 2. Specifically, by neglecting the energy of the air and the water viscosity, the total energy of the fluid is directly computed from the energy of the falling wedge in Eq. (6) as proposed in (Panciroli et al., 2015). Finally, the energy in $\widetilde{\Omega}^{*}$ is obtained by subtracting

$$
\Delta E_{\mathrm{bulk}}=E_{\mathrm{bulk}}(t)-E_{\mathrm{bulk}}(0)
$$

from $1 / 2\left(E_{\text {wedge }}(t)-E_{\text {wedge }}(0)\right)$ in Eq. (6).

\section{Numerical methods}

\subsection{Numerical modeling of the fluid flow}

The numerical simulations are performed through the interDyMFoam solver from the OpenFOAM ${ }^{\circledR}-2.2$ software package (OpenFoam, 2014), which uses the volume of fluid (VOF) method (Rider and Kothe, 1998; Scardovelli and Zaleski, 1999; Rusche, 2003; Biscarini et al., 2010) to study the incompressible flow of two immiscible fluids. According to this methodology, a single set of Navier-Stokes and continuity equations (see Eq. (1)) is solved for both phases (air and water). The interface between water and air is tracked by solving the following evolution equation for the volume fraction $\alpha$

$$
\frac{\mathrm{D} \alpha}{\mathrm{D} t}+\nabla \cdot\left[\boldsymbol{U}_{r} \alpha(1-\alpha)\right]=0
$$

The second term on the left hand side of Eq. (10) is used to reduce the diffusion of the interface (Rusche, 2003). In particular, $\boldsymbol{U}_{r}$ represents the relative velocity between the two phases. 
Equations (1) and (10), are discretized in space through the finite volume technique (Patankar, 1980). Temporal discretization, over a time step $\Delta t$, is performed through the second order accurate implicit backward scheme (OpenFOAM Foundation, 2013). The Gauss linear procedure is adopted for all the spatial interpolations, except for the convective terms, where the Gauss gamma scheme (Jasak et al., 1999) is utilized to balance numerical stability and accuracy. The discretized Navier-Stokes equations are solved through the non iterative pressure implicit with split operators (PISO) algorithm (Issa, 1985), while the discretized version of Eq. (10) is solved using the multi-dimensional universal limiter with explicit solution (MULES) procedure (OpenFOAM Foundation, 2013). All the resulting linear systems are solved through the geometric agglomerated algebraic multi grid iterative algorithm (OpenFOAM Foundation, 2013).

The fluid density and viscosity in the computational cell $i$ are calculated as the weighted average of the values corresponding to water $\left(\rho_{\mathrm{w}}\right.$ and $\left.\mu_{\mathrm{w}}\right)$ and air $\left(\rho_{\mathrm{a}}\right.$ and $\left.\mu_{\mathrm{a}}\right)$

$$
\begin{aligned}
\rho_{i}(t) & =\alpha_{i}(t) \rho_{\mathrm{w}}+\left[1-\alpha_{i}(t)\right] \rho_{\mathrm{a}}, \\
\mu_{i}(t) & =\alpha_{i}(t) \mu_{\mathrm{w}}+\left[1-\alpha_{i}(t)\right] \mu_{\mathrm{a}} .
\end{aligned}
$$

Although water and air are assumed to be incompressible, $\rho_{i}$ varies in time, as the fraction of the cell occupied by either phase may change in time. In our simulations, we set $\rho_{\mathrm{w}}=$ $1000 \mathrm{~kg} / \mathrm{m}^{3}, \rho_{\mathrm{a}}=1 \mathrm{~kg} / \mathrm{m}^{3}, \mu_{\mathrm{w}}=1 \times 10^{-3} \mathrm{Pas}$, and $\mu_{\mathrm{a}}=1.48 \times 10^{-5} \mathrm{Pas}$

The wedge is moved inside the computational domain by reallocating the mesh nodes after each time step, without changing the topology. The grid deformation is addressed using the Laplace smoothing methodology (Jasak and Tukovic, 2007; Jasak, 2008; Kassiotis, 2008).

The computational domain utilized for all the simulations is reported in Fig 3. The fluid flow is solved only for half of the physical domain, due to the problem symmetry. The overall dimensions of the computational domain are equal to $5 / 2 L \times 5 / 2 L$ and the following boundary conditions are imposed:

1. No-slip conditions and no pressure gradient along the direction orthogonal to the wall at the edges of the moving wedge ${ }^{1}$;

2. Free-slip conditions for the velocity and no-pressure gradient in the direction orthogonal to the wall along the bottom and right boundaries;

3. Relative pressure and normal velocity gradient equal to zero for the top boundary; and

4. Symmetry condition along the left boundary, so that normal velocity and the scalar fluxes across this boundary are zero.

The topology of the computational domain and the boundary conditions are schematically depicted in Fig. 3. To maximize numerical stability and accuracy, the computational domain is discretized with a block-structured mesh (Ferziger and Perić, 2002). The phases are initially at rest and the interface between them is along the $x$-axis. The pressure field is initialized according to the hydrostatic profile.

\footnotetext{
${ }^{1}$ We note that for a moving wall the condition on the pressure is in general an approximation due to the deceleration of the wedge.
} 


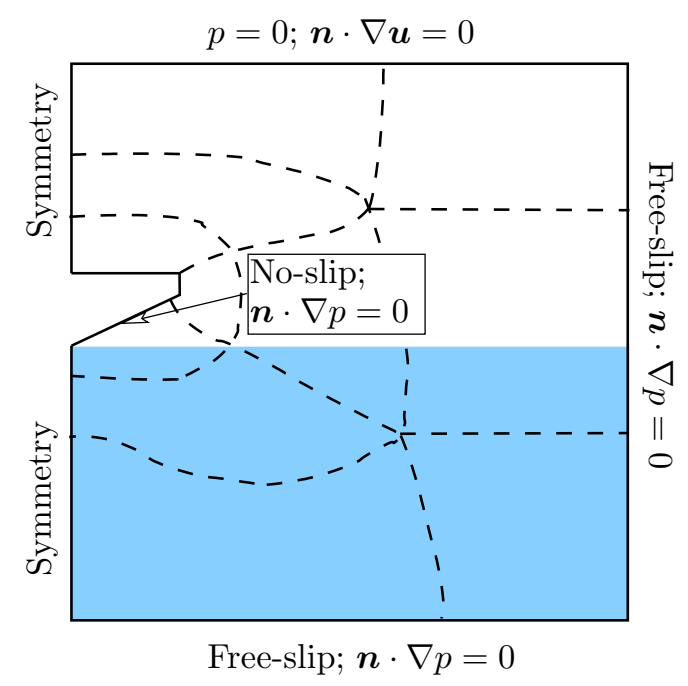

Figure 3: Representation of the mesh topology, including boundary conditions: $\boldsymbol{n}$ represents the normal to the surfaces.

4.2. Numerical estimation of the loading on the wedge and the energy transferred to the fluid

The hydrodynamic force acting on the wedge is calculated by integrating Eq. (2) with the following second order accurate formulation

$$
\boldsymbol{F}=\sum_{i=1}^{N_{\text {wedge }}} \boldsymbol{T}_{i} \boldsymbol{n}_{i} \Sigma_{i}
$$

where $N_{\text {wedge }}$ is the number of cells on the wedge surface, $\Sigma_{i}$ is the area of a generic surface element, and $\boldsymbol{n}_{i}$ is the unit vector normal to the surface element. Similarly, we estimate the mechanical energy in the fluid by integrating Eq. (5) as,

$$
E(t)=\sum_{i=1}^{N}\left[\frac{1}{2} \rho_{i}(t)\left|\boldsymbol{u}_{i}(t)\right|^{2} \Omega_{i}(t)+\rho_{i}(t) g y_{i}(t) \Omega_{i}(t)\right]
$$

where $N$ is the number of computational cells in the domain, $\Omega_{i}$ is the volume of the $i$-th cell, and $y_{i}$ is the position of the center of gravity of the cell. Notably, OpenFOAM ${ }^{\circledR}$ uses a fictitious 3-D domain (OpenFOAM Foundation, 2013) with a single cell span in the nonresolved direction so that $\Omega_{i}$ is properly defined. The work done by the wedge against the fluid is calculated by integrating Eq. (4) in time through the following second order accurate approach:

$$
W_{\text {wedge }}(t)=W_{\text {wedge }}(t-\Delta t)+\frac{F_{y}(t)+F_{y}(t-\Delta t)}{2}[\xi(t)-\xi(t-\Delta t)]
$$

where $\Delta t$ represents the numerical time step chosen so that the Courant number is lower than 0.5, to guarantee numerical stability. Note that Eqs. (12), (13), and (14) include both 
water and air; however, we consistently find that air contributes to less than $2.5 \%$ of both the force and the energy.

Beyond comparing with PIV data in (Panciroli and Porfiri, 2013), the numerical procedure described herein has been validated against experimental and theoretical results in the literature (Wagner, 1932; Zhao and Faltinsen, 1993; Zhao et al., 1996; Tveitnes et al., 2008), as discussed in AppendixA.

\section{Results and discussion}

\subsection{Comparison between CFD data and PIV findings with respect to flow kinematics and kinetics}

Here, we study the water entry of a wedge with a deadrise angle of $25^{\circ}$ dropped from a height $h_{0}$ above the water surface. Numerical simulations are performed by constraining the wedge motion to follow the experimentally tracked data by Panciroli and Porfiri (2013).

Figure 4 shows the pressure distribution predicted by the CFD simulations at time $t=$ $5 \mathrm{~ms}, 10 \mathrm{~ms}, 15 \mathrm{~ms}$, and $20 \mathrm{~ms}$, against the experimental results. The pressure is normalized as $2 p /\left(\rho_{\mathrm{w}} \dot{\xi}^{2}\right)$ and is plotted over the normalized abscissa $y / \xi$, with reference to the notations in Fig. 1. Thus, $y / \xi=-1$ identifies the keel of the wedge and positive values pertain to the pile-up. CFD and experimental data on pressure field are in good agreement along most of the wedge length. The pressure evaluated through CFD is extrapolated on the wedge surface following the same numerical scheme utilized to solve Eq. (1). The largest difference is observed in the pile-up region, where PIV data are more scarce due to light reflections that hamper the velocity measurement close to the free surface (Panciroli and Porfiri, 2013). Higher discrepancies between numerical and experimental results occur at the onset of the impact, due to the large velocity gradients and the low number of velocity vectors to perform the pressure reconstruction (Panciroli and Porfiri, 2013).

Some of these technical limitations of PIV could likely be addressed through the use of cameras affording higher resolutions and acquisition rates and considering alternative PIV schemes with interrogation regions that are tailored along the velocity gradient. Despite these limitations, PIV still offers considerable advantages with respect to pressure gauges, which only enable point measurement on the wedge and may potentially interfere with the flow, especially in the pile-up region where the spray jets are formed. The validation of PIV-based pressure reconstruction on synthetic data will further aid in the refinement of the approach and in identifying its key advantages and disadvantages.

A more comprehensive evaluation of the impact hydrodynamics is obtained from Figs. 5 and 6 that display pressure and velocity contours from simulations and experiments. While in the fluid bulk the pressure and velocity fields display similar patterns, the most significant differences between CFD and experiments are observed in the pile-up region. In fact, PIV shows a lower pile-up compared to CFD and, as a consequence, both the maximum velocity and the maximum pressure measured through PIV are lower than numerical values.

As the wedge impacts the water and decelerates, part of its energy is transferred to the fluid bulk, and to the risen water, which contributes to the formation of the pile-up and the spray jets. Figure 7 reports the energy transferred to the bulk flow as a fraction of the 


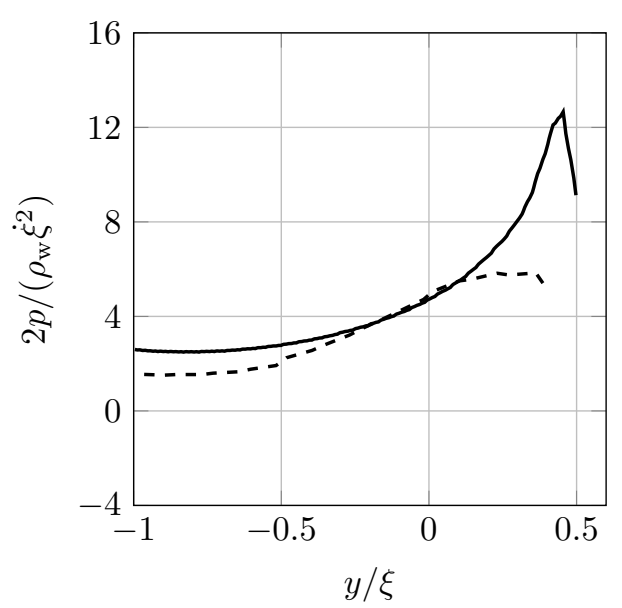

(a) $t=5 \mathrm{~ms}$

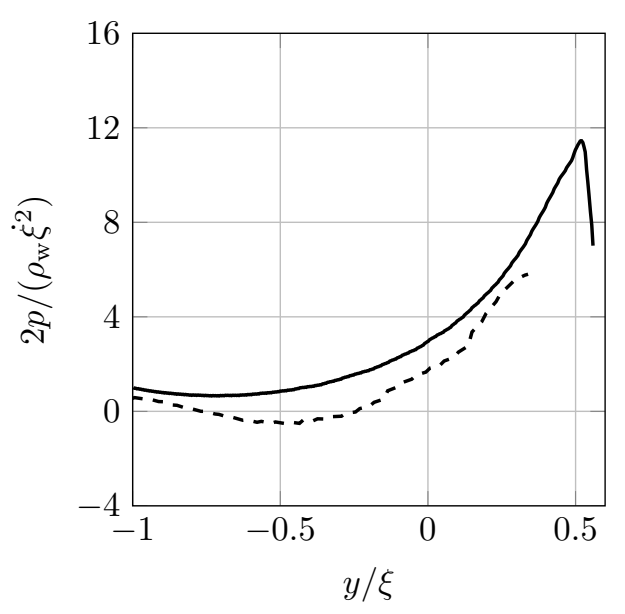

(c) $t=15 \mathrm{~ms}$

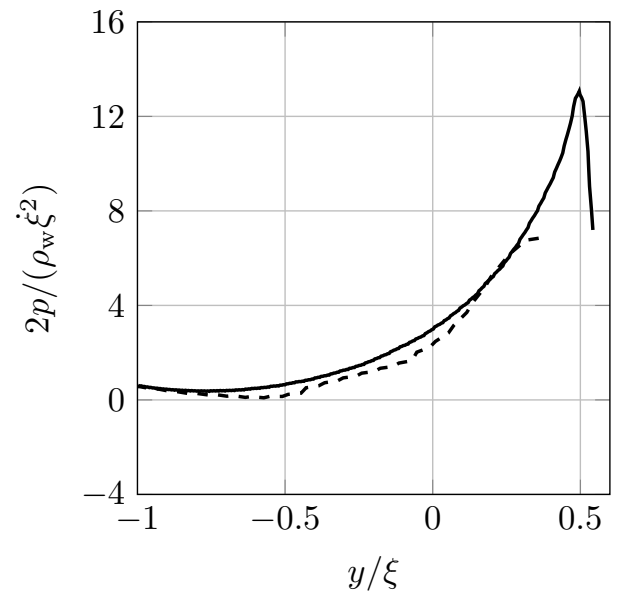

(b) $t=10 \mathrm{~ms}$

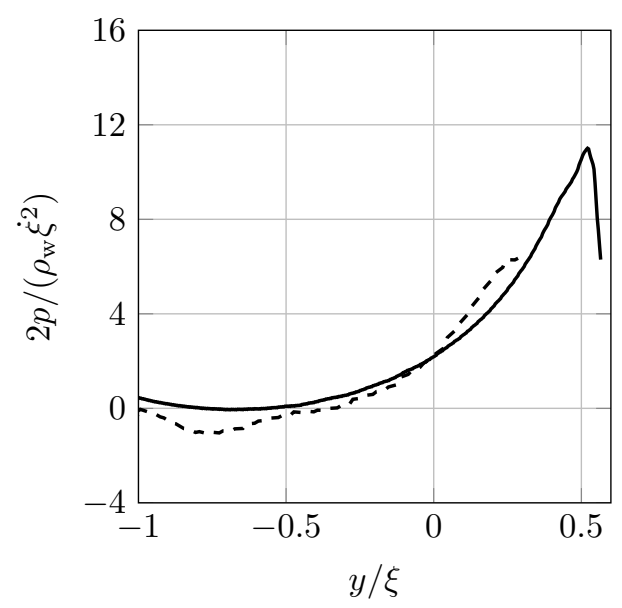

(d) $t=20 \mathrm{~ms}$

Figure 4: Normalized pressure profile over the normalized wetted surface of the wedge at several instants from the impact, for a wedge with $\beta=25^{\circ}$ falling from $h_{0}=50 \mathrm{~cm}$. Comparison between CFD results (solid lines) and experimental results in (Panciroli and Porfiri, 2013) (dashed lines).

total energy transferred to the fluid. The experimental evaluation of $W_{\text {wedge }}$ is performed through Eq. (6) on the basis of the energy, while in the numerical analysis, we use the force data via Eq. (4). Approximately $70 \%-80 \%$ of the work done by the wedge is associated with the risen water, consistent with Scolan and Korobkin (2003) who analytically studied the water entry of a three-dimensional solid body. Therein, it is shown that, for a constant entry speed, the kinetic energy is equally transmitted to the spray jets and to the rest of the water. On the other hand, according to Scolan and Korobkin (2003), when the wedge decelerates, the main part of the energy is transmitted to the jets. From Fig. 7, we also note that the portion of mechanical energy in the risen water increases with the penetration depth, which should be related to the variation of the pile-up coefficient (Panciroli et al., 
(left) CFD - (right) experimental

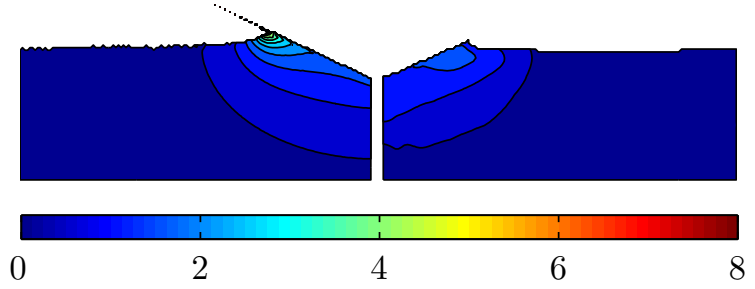

(a) $t=5 \mathrm{~ms}$

(left) CFD - (right) experimental

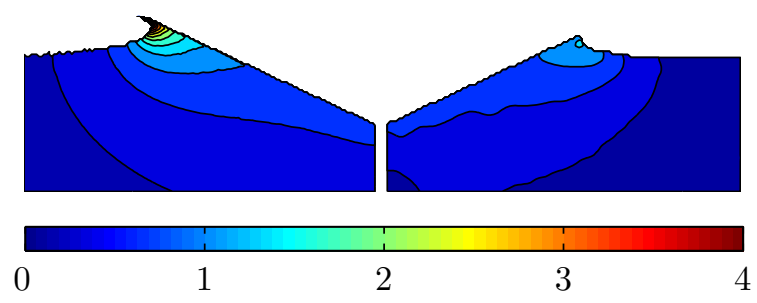

(c) $t=15 \mathrm{~ms}$ (left) CFD - (right) experimental

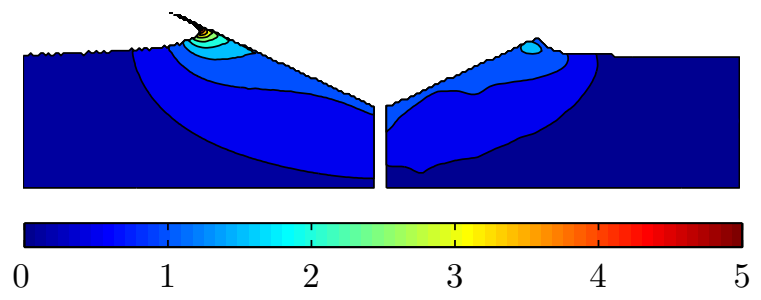

(b) $t=10 \mathrm{~ms}$

(left) CFD - (right) experimental

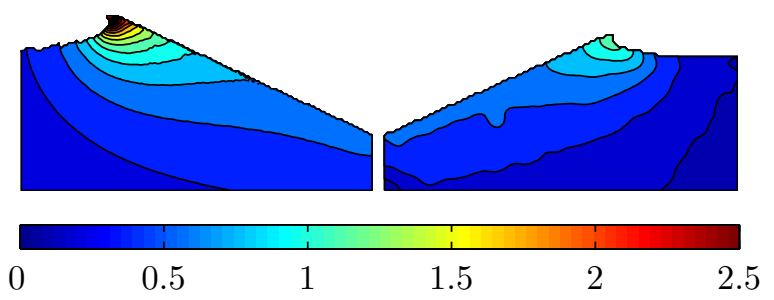

(d) $t=20 \mathrm{~ms}$

Figure 5: Comparison between numerical and experimental velocity fields for a wedge with $\beta=25^{\circ}$ falling from $h_{0}=50 \mathrm{~cm}$. The color-bar represents the fluid velocity magnitude expressed in $\mathrm{m} / \mathrm{s}$.

2015).

Figure 8 offers further comparison between experimental and CFD data in terms of the pile-up coefficient $\phi$. In the numerical simulations, the water-air interface is diffused over $2-3$ rows of cells and therefore does not have a uniquely defined position. Thus, we utilize the location of the peak pressure to identify the pile-up front, which is likely to slightly underestimate the pile-up coefficient. However, such a difference should be negligible compared to experimental uncertainties, as the pressure decay after the peak is very rapid, as illustrated in AppendixA for example. Although the numerical values for $\phi$ are about $25 \%$ higher compared to experimental results from (Panciroli et al., 2015), in both CFD and PIV the pile-up coefficient increases with the wedge penetration depth. This aspect is particularly relevant since most of the analytical models for water entry problems consider a constant pile-up coefficient. For example Wagner (1932) has predicted a coefficient of $\pi / 2$, and others have found lower values (Mei et al., 1999) as function of the deadrise angle but independent of the entry depth.

\subsection{Assessment of the pressure reconstruction methodology through CFD dataset}

Here, we utilize the CFD dataset to verify our PIV-based pressure reconstruction, and quantify the influence of the relevant PIV parameters on accuracy. At first, CFD data are interpolated over a grid of $140 \times 70$ points with the original time-step $\Delta t=0.25 \mathrm{~ms}$ to construct our synthetic dataset and verify the PIV-based pressure reconstruction. Then, a parametric analysis is performed by varying the vector grid spacing and the time-step. CFD 
(left) CFD - (right) reconstructed pressure

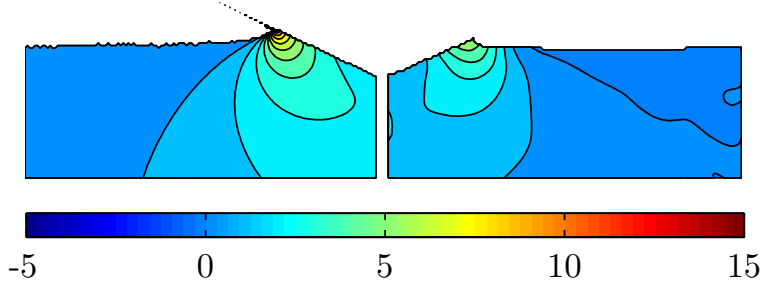

(a) $t=5 \mathrm{~ms}$

(left) CFD - (right) reconstructed pressure
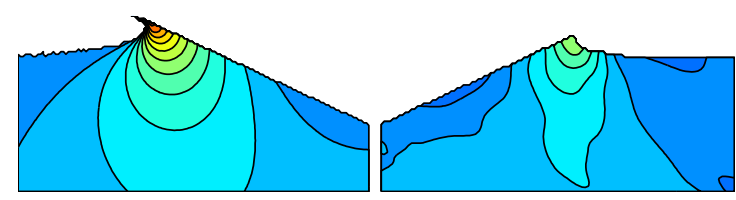

$-5$

0 (left) CFD - (right) reconstructed pressure

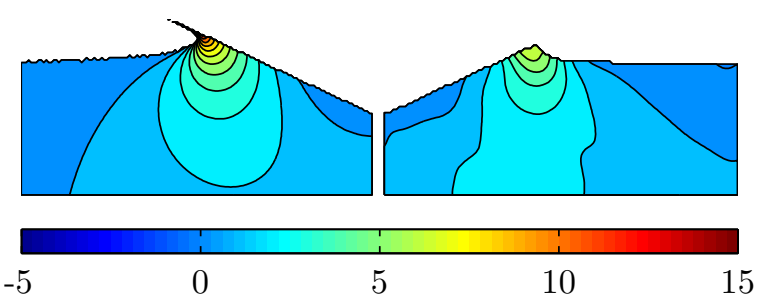

(b) $t=10 \mathrm{~ms}$

(left) CFD - (right) reconstructed pressure

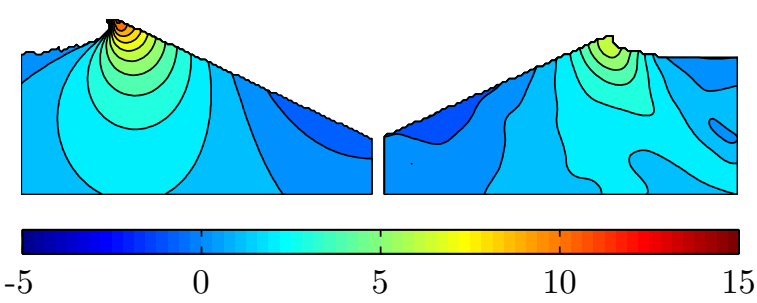

(d) $t=20 \mathrm{~ms}$

Figure 6: Comparison between numerical and experimental normalized pressure fields for a wedge with $\beta=25^{\circ}$ and falling from $h_{0}=50 \mathrm{~cm}$. Pressure is normalized as $2 p /\left(\rho_{\mathrm{w}} \dot{\xi}^{2}\right)$.

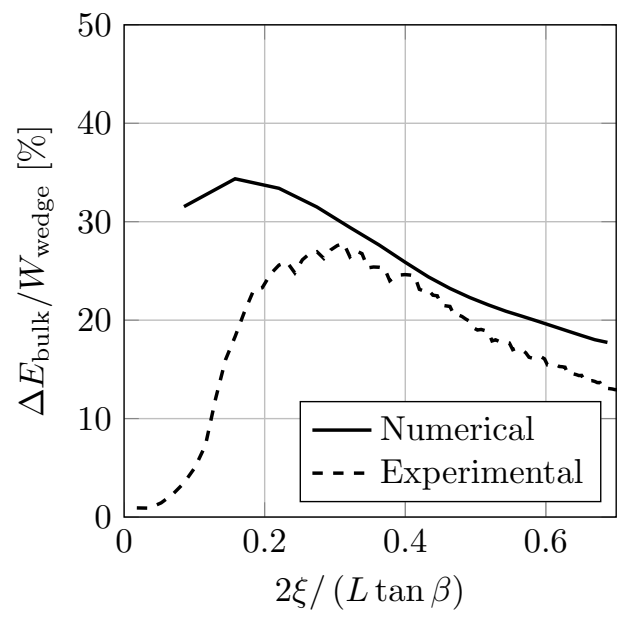

Figure 7: Mechanical energy imparted to the bulk flow as a fraction of the total energy transferred to the fluid, for a wedge with $\beta=25^{\circ}$ falling from $h_{0}=50 \mathrm{~cm}$. $\Delta E_{\text {bulk }}$ is defined in Eq. (9).

simulations cover a fluid domain that is $500 \mathrm{~mm}$ in length and $250 \mathrm{~mm}$ in depth. Thus, to reproduce a domain similar to the camera view in (Panciroli and Porfiri, 2013), CFD data are cropped to a region $130 \mathrm{~mm}$ large and $64 \mathrm{~mm}$ deep.

Figure 9 displays a comparison between CFD results and pressure values reconstructed through the methodology proposed by Panciroli and Porfiri (2013). For ease of illustration 


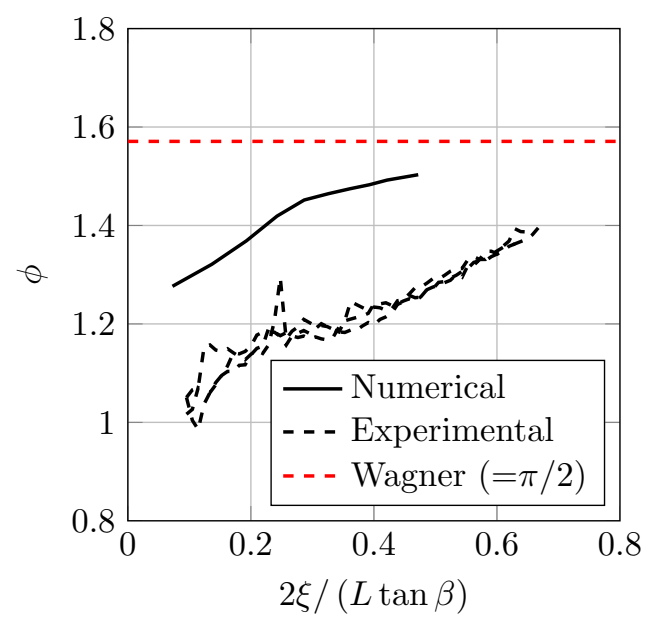

Figure 8: Comparison between experimental data on the pile-up coefficient and numerical predictions for a decelerating wedge with $\beta=25^{\circ}$ falling from $h_{0}=25 \mathrm{~cm}$. Experimental data are from (Panciroli et al., 2015)

(left) CFD - (right) reconstructed pressure

(left) CFD - (right) reconstructed pressure
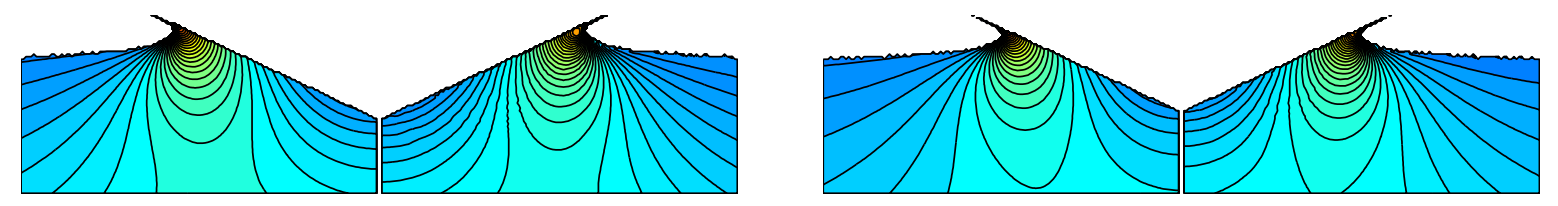

$-5$

0

$5 \quad 10$

15

(a) $t=5 \mathrm{~ms}$
$-5$

0
10

15

(b) $t=10 \mathrm{~ms}$

(left) CFD - (right) reconstructed pressure

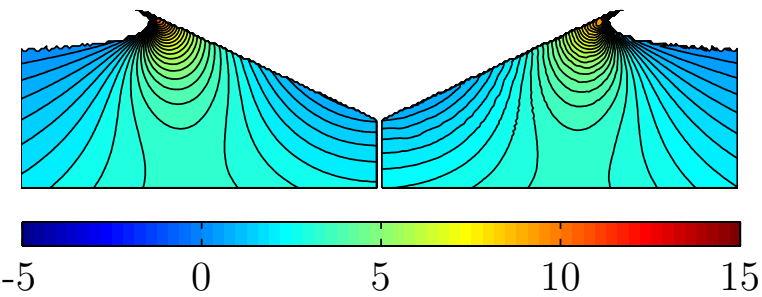

(c) $t=15 \mathrm{~ms}$ (left) CFD - (right) reconstructed pressure

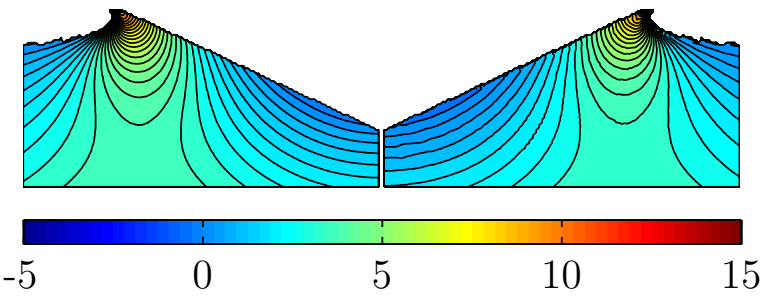

(d) $t=20 \mathrm{~ms}$

Figure 9: Comparison between CFD results (left) and pressure reconstruction from such a synthetic dataset (right) for the normalized hydrodynamic pressure after (a) $5 \mathrm{~ms}$, (b) $10 \mathrm{~ms}$, (c) $15 \mathrm{~ms}$, and (d) $20 \mathrm{~ms}$ from the impact.

CFD data are mirrored about the symmetry axis. The reconstructed pressure is in very good agreement with CFD data in the whole domain. In Fig. 10, we detail such a comparison 


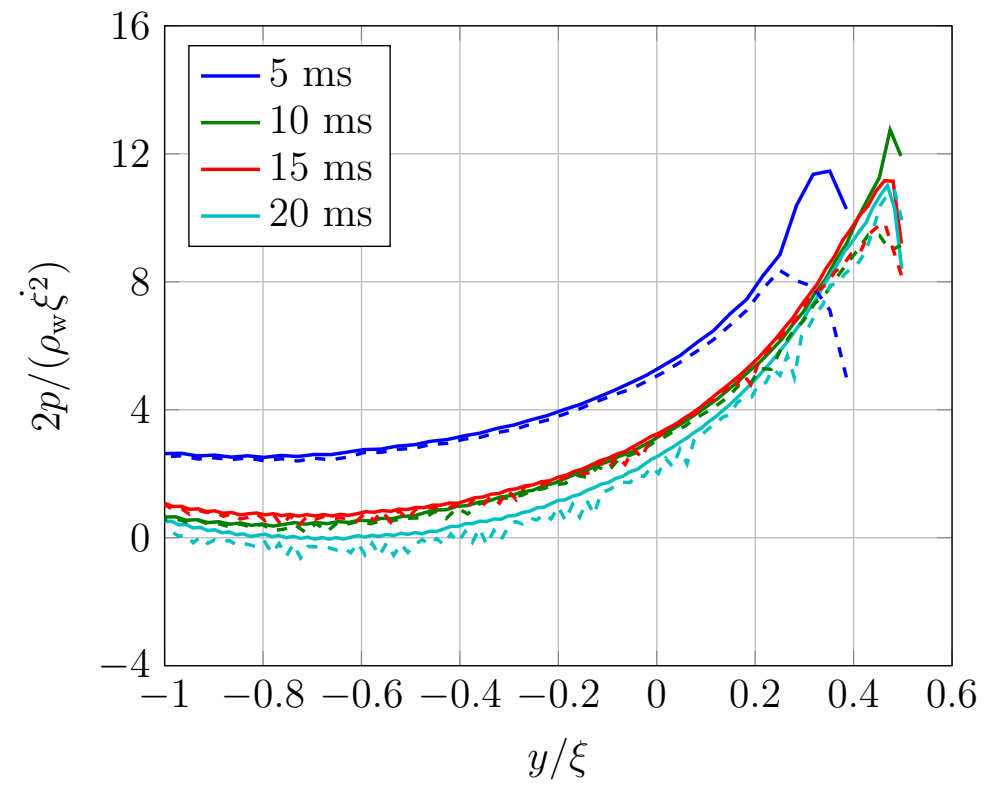

Figure 10: Comparison between CFD results (solid lines) and pressure reconstruction from such a synthetic dataset (dashed lines) for the normalized hydrodynamic pressure on the wetted surface of the wedge at several instants.

along the wetted surface of the wedge to elucidate the hydrodynamic loading. We remark that CFD results do not refer to the original grid of the numerical model, but to the downsampled grid of the synthetic dataset. Thus, CFD data do not yield the exact pressure on the wetted surface of the body, but they rather identify the theoretical value that PIV-based reconstruction should ideally predict. In fact, as the data are down-sampled to a uniformly spaced grid, grid points do not perfectly align on the fluid-structure interface. Notably in a real experiment, as shown in Fig. 4, additional factors limit the accuracy of PIV-based reconstruction, including light reflections at the free surface, uncertainties in masking the moving wedge, and inaccuracies in estimating the velocity field due to large velocity gradients at the onset of the impact.

PIV-based pressure reconstruction is accurate in estimating the flow kinetics for the considered synthetic dataset. However, as discussed by Panciroli and Porfiri (2013), in the early stage of the impact the reconstructed maximum pressure is lower than expected. Such behavior could be ascribed to the limited number of vectors over which the pressure is reconstructed. As time advances, the pile-up region increases in size, resulting in a larger number of vectors. Thus, as time progresses the peak value is correctly reconstructed.

\subsubsection{Influence of the vector grid spacing}

Here, we repeat the same analysis parametrically varying the grid spacing of the synthetic dataset used in the PIV-based reconstruction. CFD data are down-sampled for different grids to assess the influence of PIV image resolution on pressure reconstruction. In particular, increasing the spacing of the grid, we simulate PIV images with a lower resolution. In 
the following, we refer to the grid spacing as $\mathcal{R}_{n}$, where $n$ is a multiplier for the base vectors grid of $20 \times 10$. Specifically, $n=7$ corresponds to the original grid spacing in (Panciroli and Porfiri, 2013), discussed earlier, that is, $140 \times 70$, and $n=10$ to the maximum refinement, corresponding to a grid $200 \times 100$. The parametric analysis is performed utilizing $n=1,2, \ldots, 10$.
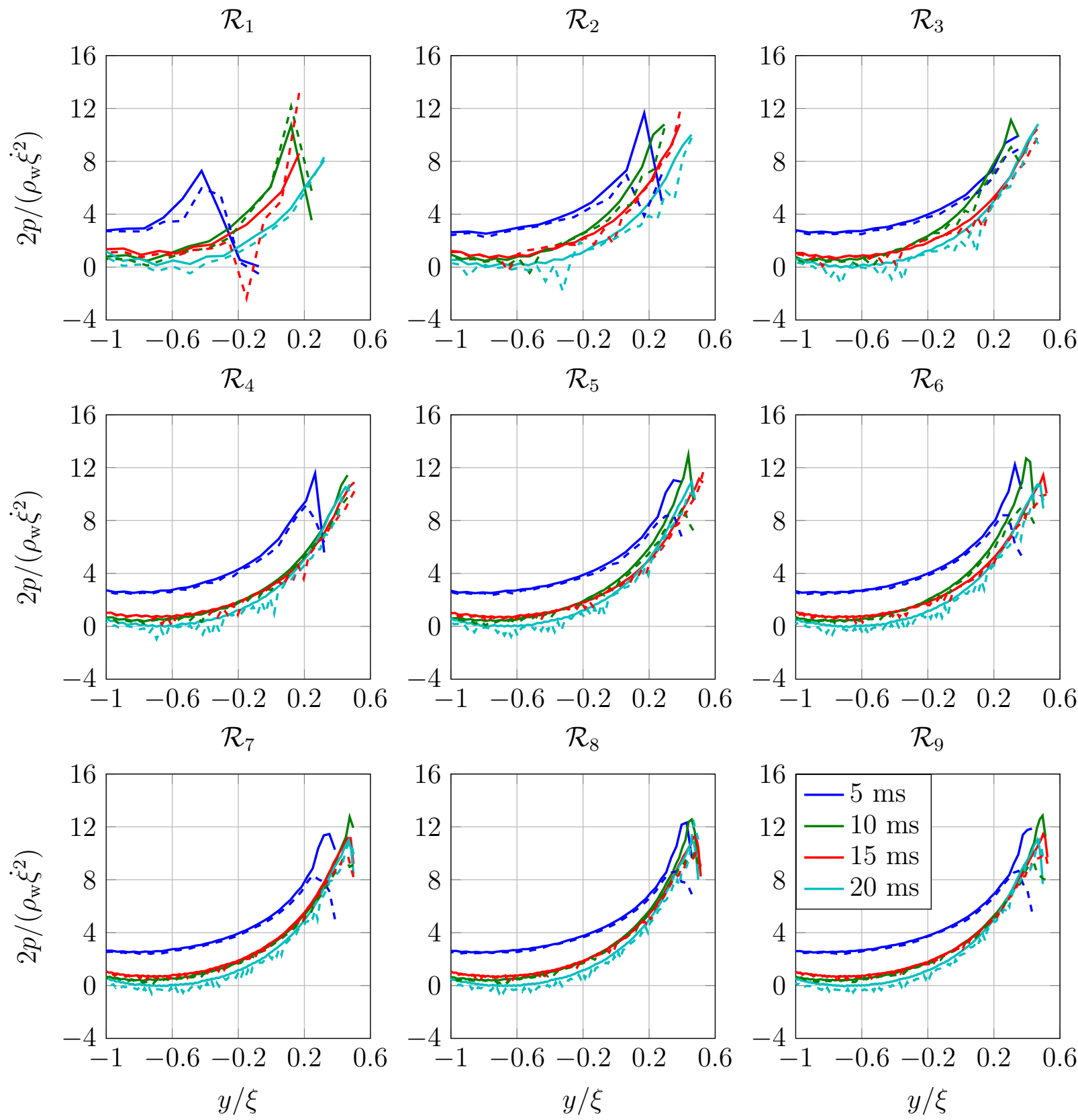

Figure 11: Comparison between CFD results (solid lines) and pressure reconstruction from such a synthetic dataset (dashed lines) for the normalized hydrodynamic pressure on the wetted surface of the wedge at several instants and for different grid refinements. 
Figure 11 compares synthetic data and reconstructed pressure profiles on the wetted surface of the wedge for varying vector grid refinements. Surprisingly, our results indicate that the PIV-based reconstruction is successful even for the coarser grid $\mathcal{R}_{1}$. The major drawback of utilizing coarse grids is the large distance between the location of the grid points and the actual fluid-structure interface. While PIV-based reconstruction is successful in anticipating the synthetic data, such a dataset is a poor description of the pressure on the wetted surface of the wedge. Moreover, results from $\mathcal{R}_{1}$ indicate that a low image resolution is associated with a loss of information close to the pile-up, which, in turn, causes an underestimation of the maximum pressure. The distance of the actual fluid-structure interface from the measured values on the grid points is shown in Fig. 12, for the refinement $\mathcal{R}_{2}$ after $15 \mathrm{~ms}$ from the impact. Therein, the fluid-structure interface and the water free surface are shown as continuous black lines.

(left) CFD - (right) reconstructed pressure

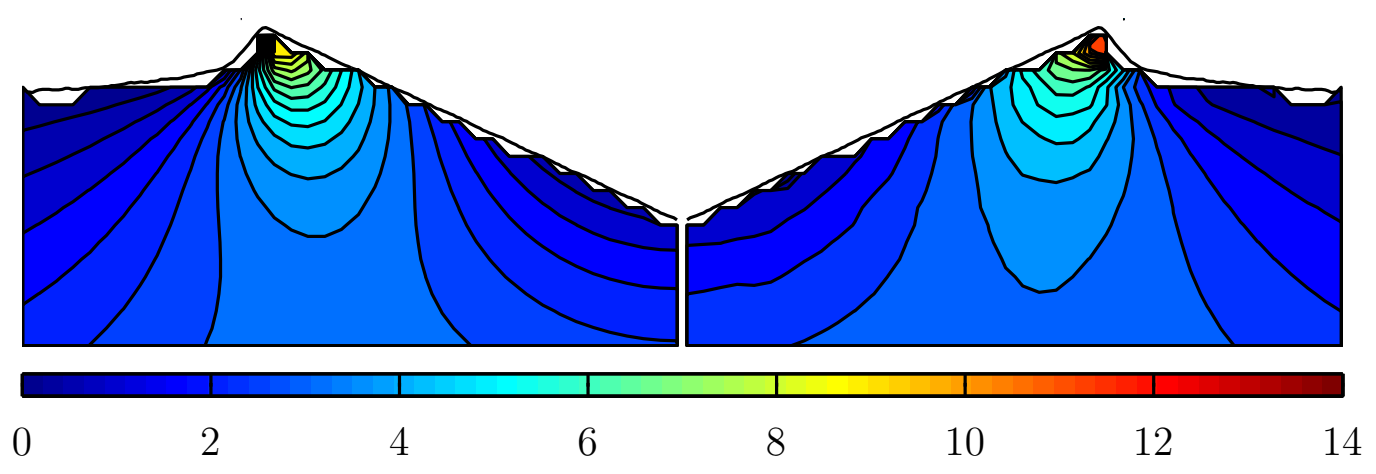

Figure 12: Comparison between CFD results (left) and pressure reconstruction from such a synthetic dataset (right) for the normalized hydrodynamic pressure for the grid refinement $\mathcal{R}_{2}$ after $15 \mathrm{~ms}$ from the impact. The fluid-structure interface and the water free surface are marked as solid black lines.

The accuracy of the results seems to converge for refinements greater than $\mathcal{R}_{6}$. However, at $t=5 \mathrm{~ms}$, the peak pressure in the pile-up is underestimated by the PIV-based reconstruction for all the refinements. Such a behavior is ascribed to the underestimation of the fluid acceleration in the pile-up at the onset of the impact and should thus be related to the limited acquisition frequency of $4 \mathrm{kHz}$.

A quantitative assessment of the quality of the reconstructed results can be obtained through an error analysis. For each grid refinement, and at each time $t$, we score the error between the synthetic dataset and the reconstructed pressure as

$$
\varepsilon(t)=\frac{1}{N_{\mathrm{int}}} \sum_{j=1}^{N_{\mathrm{int}}} \frac{\left|p_{\mathrm{CFD}}^{j}(t)-p_{\mathrm{PIV}}^{j}(t)\right|}{\max \left(p_{\mathrm{CFD}}(t)\right)-\min \left(p_{\mathrm{CFD}}(t)\right)},
$$

where $N_{\text {int }}$ is the total number of grid points that are adjacent to the fluid-structure interface, $p_{\mathrm{CFD}}^{j}$ is the pressure at point $j$ from CFD, and $p_{\text {PIV }}^{j}$ is the corresponding reconstructed 
pressure.

For all the ten refinements, we find that the mean error in the window [2 ms, $20 \mathrm{~ms}]$, defined as $\bar{\varepsilon}=\frac{1}{72} \sum_{k=0}^{71} \varepsilon(2 \mathrm{~ms}+k \Delta t)$, where 72 is the total number of time-steps, is always below $3 \%$, with a maximum standard deviation $\sigma=\sqrt{\frac{1}{71} \sum_{k=0}^{71}(\varepsilon(2 \mathrm{~ms}+k \Delta t)-\bar{\varepsilon})^{2}}$ of $1.56 \%$. These results, summarized in Table 1, confirm the feasibility of PIV-based pressure reconstruction across a wide range of grid resolutions.

Table 1: Mean error $\bar{\varepsilon}$ and standard deviation $\sigma$ between CFD and PIV data, both evaluated at the synthetic grid points and for the whole duration of the impact.

\begin{tabular}{|l|c|c|}
\hline & $\bar{\varepsilon}[\%]$ & $\sigma[\%]$ \\
\hline \hline $\mathcal{R}_{1}$ & 2.95 & 1.56 \\
$\mathcal{R}_{2}$ & 2.37 & 1.36 \\
$\mathcal{R}_{3}$ & 1.93 & 1.03 \\
$\mathcal{R}_{4}$ & 1.73 & 0.95 \\
$\mathcal{R}_{5}$ & 1.59 & 0.85 \\
$\mathcal{R}_{6}$ & 1.60 & 0.77 \\
$\mathcal{R}_{7}$ & 1.60 & 0.76 \\
$\mathcal{R}_{8}$ & 1.58 & 0.68 \\
$\mathcal{R}_{9}$ & 1.65 & 0.72 \\
$\mathcal{R}_{10}$ & 1.65 & 0.69 \\
\hline
\end{tabular}

Table 2: Mean error $\bar{\varepsilon}$ and standard deviation $\sigma$ between CFD pressure evaluated on the fluid structure interface and PIV data evaluated at the closest point on the synthetic grid, for the whole duration of the impact.

\begin{tabular}{|l|c|c|}
\hline & $\bar{\varepsilon}[\%]$ & $\sigma[\%]$ \\
\hline \hline $\mathcal{R}_{1}$ & 11.06 & 5.04 \\
$\mathcal{R}_{2}$ & 8.19 & 3.36 \\
$\mathcal{R}_{3}$ & 6.02 & 2.06 \\
$\mathcal{R}_{4}$ & 4.79 & 1.77 \\
$\mathcal{R}_{5}$ & 3.74 & 1.55 \\
$\mathcal{R}_{6}$ & 3.10 & 1.22 \\
$\mathcal{R}_{7}$ & 2.62 & 1.22 \\
$\mathcal{R}_{8}$ & 2.31 & 1.11 \\
$\mathcal{R}_{9}$ & 2.07 & 0.97 \\
$\mathcal{R}_{10}$ & 1.65 & 0.69 \\
\hline
\end{tabular}

The error between CFD data and the reconstructed pressure is very low for all the grid refinements when both CFD and reconstructed data refer to the same grid points, as shown in Table 1. On the other hand, if we compare the reconstructed pressure with actual CFD findings on the wedge surface, the error, presented in Table 2, is higher than $10 \%$ with a standard deviation of $5 \%$ for the coarser grid. However, such an error decreases below $2 \%$ 
for the more refined grid, since grid points approach the fluid-structure interface as the grid refinement improves.

An error of $10 \%$ between CFD and PIV-based reconstruction should be considered acceptable given the complexity of the problem and technical limitations of PIV technology. However, we should acknowledge that in coarser grids the limited number of vectors in the pile-up region hampers the possibility of capturing the pressure peak. In fact, grid points might be far from the maximum pressure location, thus leading to an underestimation of the hydrodynamic loading experienced by the wedge during water entry.

\subsubsection{Influence of the sampling frequency}

Here, we study the effect of the time-step on the accuracy of the PIV-based pressure reconstruction methodology. The analysis is performed on grid $\mathcal{R}_{7}$, corresponding to the setup used by Panciroli and Porfiri (2013). The time-step is varied according to acquisition frequencies of $4 \mathrm{kHz}, 2 \mathrm{kHz}, 1 \mathrm{kHz}$, and $0.5 \mathrm{kHz}$, where $4 \mathrm{kHz}$ corresponds to the CFD timestep. Thus, in the synthetic data, we retain one time sample every eight, four, or two as we subsample the CFD dataset at $0.5 \mathrm{kHz}, 1 \mathrm{kHz}$, or $2 \mathrm{kHz}$.
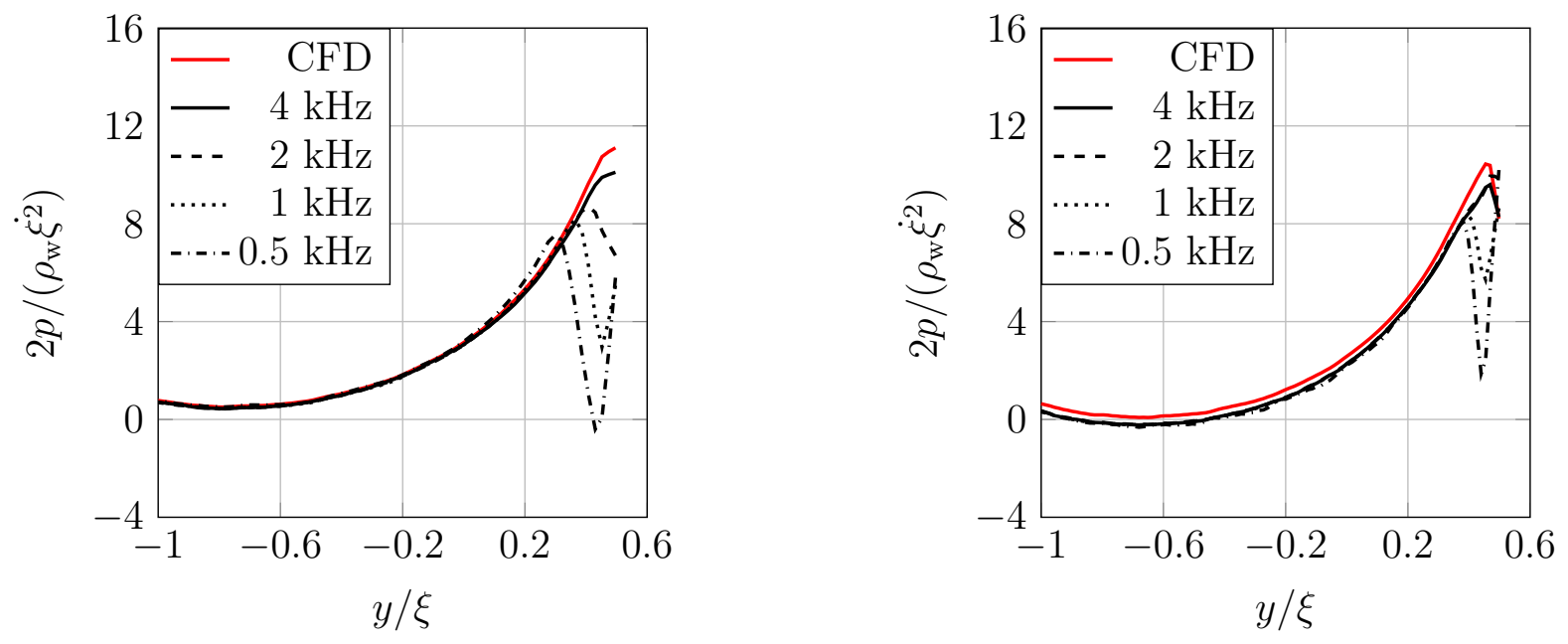

Figure 13: Comparison between CFD results (black lines) and pressure reconstruction from such a synthetic dataset (red lines) for the normalized hydrodynamic pressure on the wetted surface of the wedge for different sampling frequencies at two instants: $t=10 \mathrm{~ms}$ (left) and $t=20 \mathrm{~ms}$ (right)

A comparison between the pressure reconstruction for different sampling frequencies and the pressure computed through CFD is reported in Fig. 13 in terms of normalized hydrodynamic pressure along the wetted surface. As the time-step increases, the pressure peak decreases and its position moves away from the free surface. Such a behavior partially justifies the differences between CFD data and reconstructed results observed for all the grid refinements at the onset of the impact, as shown in Fig. 11. The underestimation of the pressure peak is more severe for earlier times, since the acceleration in the pile-up is maximized at the onset of the impact. On the other hand, the reconstructed pressure at the keel is not influenced by the sampling frequency. 


\section{Conclusions}

In this paper, we propose the use of CFD to assess the validity of a PIV-based experimental methodology for the analysis of water entry problems. The computational framework is based on the volume of fluid model, in which water and air are treated as immiscible, a single set of Navier-Stokes and continuity equations is solved for both phases and the free surface is tracked through the evolution of the fluid volume fraction. We focus on the water entry of a rigid wedge with a deadrise angle of $25^{\circ}$ in free fall from different heights.

A close comparison between CFD and experimental data in terms of the pressure and velocity fields supports the validity of the proposed approach for studying water entry problems. Discrepancies between numerical and experimental results are seen only in a region close to the free surface, due the unavoidable light reflections in the PIV images that preclude to reconstruct the velocity field in a thin layer close to the free surface. We also study the flux of mechanical energy established between the falling wedge and the fluid flow, confirming that about $70 \%-80 \%$ of the work done by the falling wedge is imparted to the risen water, comprising the pile-up and the spray jets. Further, we find that the pile-up coefficient increases with the penetration of the wedge, while being well below the Wagner's theory.

The main contribution of this work is the assessment of the PIV-based approach to reconstruct the pressure on synthetic dataset generated through CFD. This synthetic dataset is processed though the pressure reconstruction methodology proposed by Panciroli and Porfiri (2013). Results demonstrates that the reconstruction methodology is robust with respect to variations of the vector grid spacing. Specifically, we find that the reconstructed pressure is extremely well in line with the pressure of the synthetic dataset, independently of the grid spacing. However, coarse grid spacing leads to less accurate estimates of the pressure peak, whereby the pressure tends to rapidly vary along the wetted surface of the wedge. The sensitivity analysis on the camera acquisition rate in the range $0.5-4 \mathrm{kHz}$ demonstrates that the pressure peak could be significantly underpredicted for low (lower than $1 \mathrm{kHz}$ ) acquisition frequency. Such an error decreases in time as the wedge penetrates the water surface, reducing its acceleration. On the other hand, in vicinity of the keel, where more moderst accelerations are attained, the reconstructed pressure is nearly independent of the acquisition frequency.

\section{Acknowledgements}

This work is supported by the Office of Naval Research grant N00014-10-1-0988, with Dr. Y. D. S. Rajapakse as the program manager, and by the Italian Research Project MISEICE-CRUI n. 55 2010, "Metodologia integrata teorico-numerico-sperimentale per l'analisi fluido-struttura nel settore navale". Views expressed herein are those of authors and not of the funding agencies. 


\section{AppendixA. Grid sensitivity analysis and validation of the numerical fluid dy- namics model}

Here, we focus on the impact of a rigid V-shaped wedge with a deadrise angle $\beta=15^{\circ}$ and moving with constant downward speed $\dot{\xi}=1 \mathrm{~m} / \mathrm{s}$. In order to establish grid independence, we performed the same numerical simulation with the three different computational meshes shown in Fig. A.14, which differ from one another as follows:

1. in Grid 1, the maximum wetted surface of the wedge is discretized using 125 elements, and the wedge keel is initially placed at $y=0$ (see Fig A.14(a));

2. in Grid 2, the maximum wetted surface of the wedge is discretized using 250 elements, and the wedge keel is initially placed at $y=0$ (see Fig A.14(b)); and

3. in Grid 3, the maximum wetted surface of the wedge is discretized using 125 elements, and the wedge keel is initially placed at $y=2 L$ (see Fig A.14(c)).

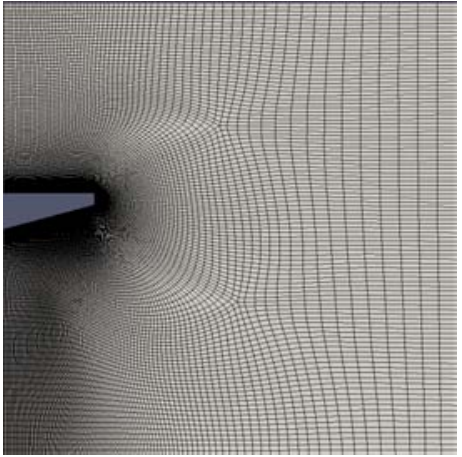

(a) Grid 1

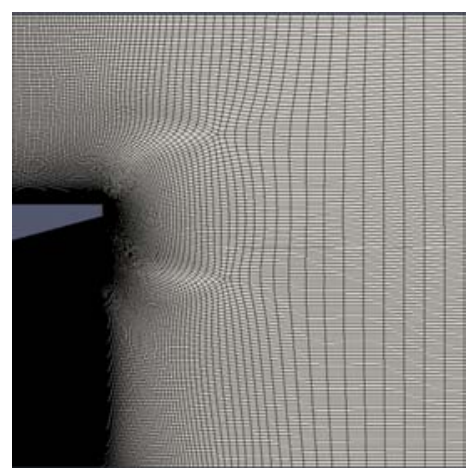

(b) Grid 2

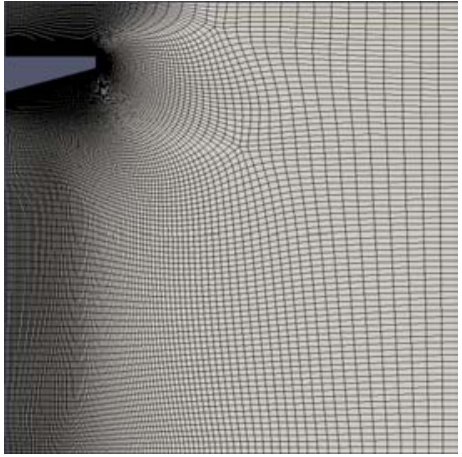

(c) Grid 3

Figure A.14: Computational meshes used for the grid sensitivity analysis.

Comparing the results obtained with Grid 1, Grid 2, and Grid 3, it is possible to assess the effects of the mesh density, and of the distance between the external domain boundaries from the moving wedge. As shown in Fig. A.15(a), the vertical force is not significantly influenced by the grid refinement neither by the distance between the wedge and the bottom wall. Figure A.15(a) also evidences that the hydrodynamic force acting on the hull increases almost linearly with time, in agreement with the analytical models of Wagner (Wagner, 1932) and Von Karman (Von Karman, 1929). As expected, the force per unit width from the simulations is bounded above by the analytical prediction of Wagner theory (Wagner, 1932)

$$
F_{y_{\mathrm{Wa}}}=\rho_{\mathrm{w}} \frac{\pi^{2} r_{\mathrm{Wa}} \dot{\xi}^{2}}{4 \tan \beta}
$$

and below by von Karman theory (Von Karman, 1929)

$$
F_{y_{\mathrm{VK}}}=\rho_{\mathrm{w}} \frac{\pi r_{\mathrm{VK}} \dot{\xi}^{2}}{2 \tan \beta},
$$


where $r_{\mathrm{Wa}}=\pi \xi /(2 \tan \beta)$, and $r_{\mathrm{VK}}=\xi / \tan \beta$ are the wetted length estimated by Wagner (1932) and Von Karman (1929), respectively. The pressure profile on the wetted surface of the wedge is estimated using the three grids reported in Fig. A.15(b), and compared to the Wagner theory (Wagner, 1932)

$$
p_{\mathrm{Wa}}=\rho_{\mathrm{w}}\left[\frac{\pi}{2} \frac{\dot{\xi}^{2} r_{\mathrm{Wa}}}{\tan \beta \sqrt{r_{\mathrm{Wa}}^{2}-x^{2}}}-\frac{1}{2} \frac{\dot{\xi}^{2} x^{2}}{r_{\mathrm{Wa}}^{2}-x^{2}}\right] .
$$

The grid refinement from Grid 1 to Grid 2 slightly increases the pressure peak of about $17 \%$. Yet, differences are well below 1\% along most of the wedge except for a region composed by two computational cells.

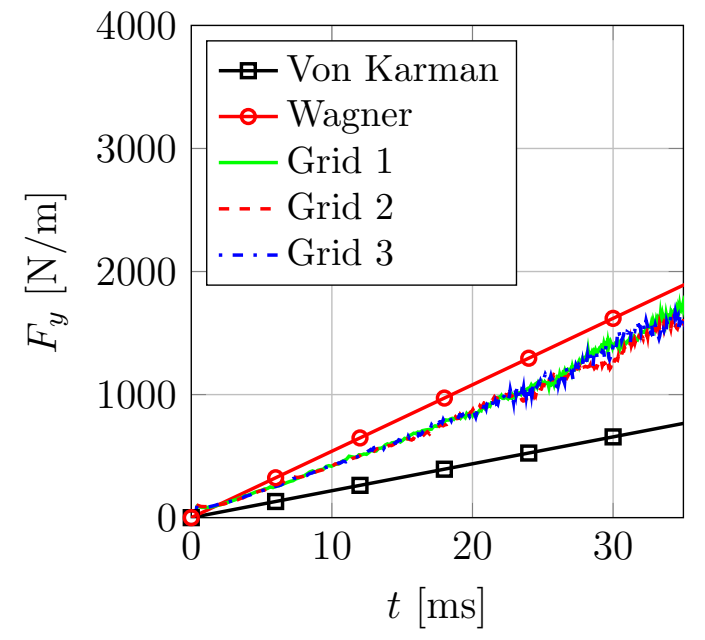

(a) Vertical force

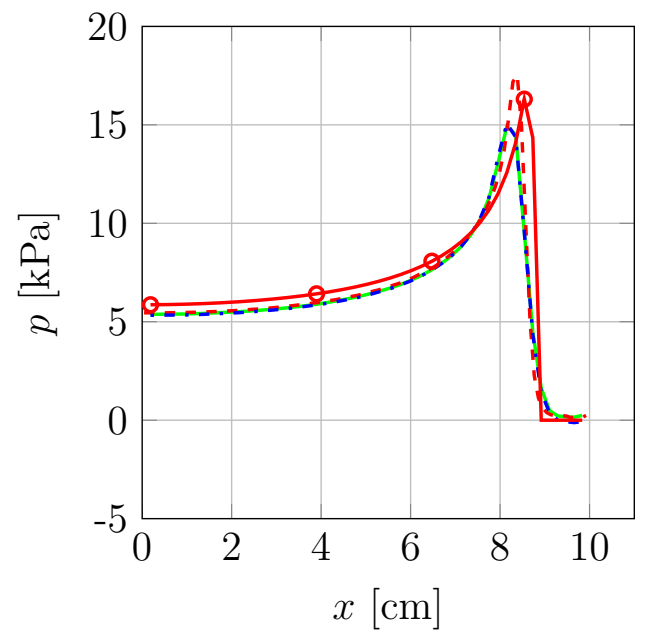

(b) Pressure profile

Figure A.15: Influence of mesh density and overall dimension on the vertical force and on the pressure profile on the wedge.

In order to test the numerical setup for energy conservation, in Fig. A.16 we compare the work done by the wedge with the variation of the mechanical energy in the whole computational domain:

$$
\Delta E(t)=E(t)-E(0) .
$$

Our results indicate that the mechanical energy is conserved for all three grids, and that our numerical scheme effectively captures the energy flow from the moving wedge to the fluid. In fact, at the end of the simulation, the difference between $W_{\text {wedge }}$ and $\Delta E$ is less than $5 \%$ for all grids. We noted that viscous dissipation is expected to be almost negligible for an impulsive phenomenon such as the water impact of a blunt body. In fact, due to the short duration of the event, mechanical energy is not transmitted from the large length scales (on the order of magnitude of $L$ ), to the smallest length scales pertaining to viscosity (Seng et al., 2012). 


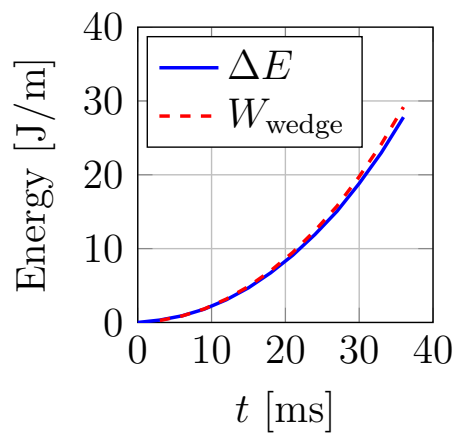

(a) Grid 1

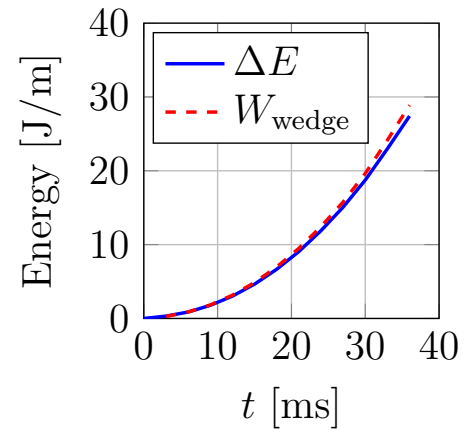

(b) Grid 2

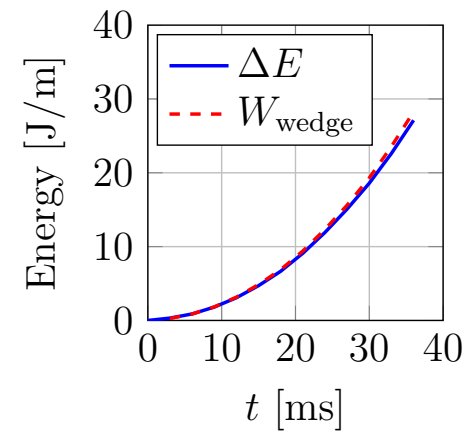

(c) Grid 3

Figure A.16: Influence of mesh density and overall dimension on mechanical energy conservation.

Mass conservation is also verified for all the performed simulations, and we confirm that the maximum mass variation in the simulation domain is less than $10^{-4 \%}$. Thereafter, all the simulations in the results section are based on Grid 1, considered as a good compromise between numerical accuracy and computational efficiency.

A further insight on the reliability of the proposed numerical scheme is possible through comparison of the calculated hydrodynamic pressure with the analytical formulation of Wagner (1932) given by Eq. A.3. Figure A.17 illustrates the pressure distribution predicted by the proposed method for a wedge entering the water at a constant speed $\dot{\xi}=1 \mathrm{~m} / \mathrm{s}$, with $\beta$ varying in the range $15^{\circ}-40^{\circ}$. In line with the hypothesis of small deadrise angles underlying Wagner's theory (Wagner, 1932; Faltinsen, 2005), the difference between numerical and theoretical pressure profiles is negligible for $\beta<25^{\circ}$, while, for larger $\beta$ the numerical pressure gradually deviates from the analytical one. From numerical simulations, the minimum of the pressure is not attained at the wedge keel for $\beta \geq 35^{\circ}$, in contrast with Wagner's formulation. Such a pressure profile is qualitatively confirmed by direct measurements, analytical, and numerical results by Zhao and Faltinsen (1993); Zhao et al. (1996), and Yettou et al. (2007). Specifically, in (Zhao and Faltinsen, 1993; Zhao et al., 1996), an extension of the low deadrise angle approximation is proposed. In (Zhao et al., 1996), it is also confirmed that the minimum of the pressure is not located at the wedge keel for $\beta$ greater than $40^{\circ}$.

The validity of the numerical procedure is also assessed by comparing numerical findings with the experimental results by Tveitnes et al. (2008). Therein, a V-shaped hull enters calm water with constant velocity and the vertical forces acting on different sections of the wedge are measured. We simulate the impact of two wedge samples, with deadrise angles equal to $15^{\circ}$ and $30^{\circ}$, respectively. Both samples have a length $L=0.6 \mathrm{~m}$, a width of $0.3 \mathrm{~m}$, and a penetration velocity $\dot{\xi}=0.94 \mathrm{~m} / \mathrm{s}$. The time evolution of the hydrodynamic force acting on the hull is shown in Fig. A.18, where numerical results obtained by multiplying $F_{y}$ by the sample width are compared with experiments. Numerical findings are in good agreement with experimental data, especially for $\beta=30^{\circ}$. However the vertical component of the hydrodynamic force is overestimated by the numerical solution for $\beta=15^{\circ}$. This should be 


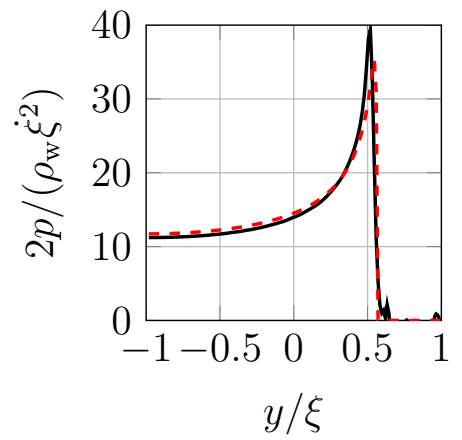

(a) $\beta=15^{\circ}$

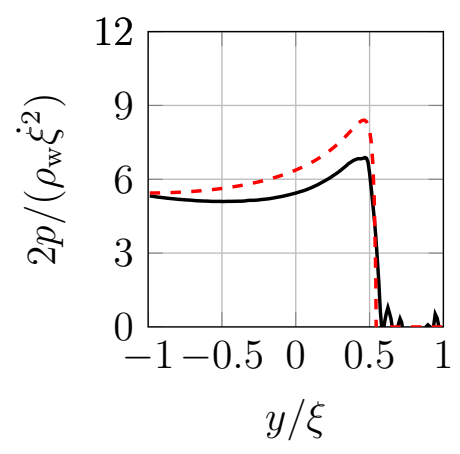

(d) $\beta=30^{\circ}$

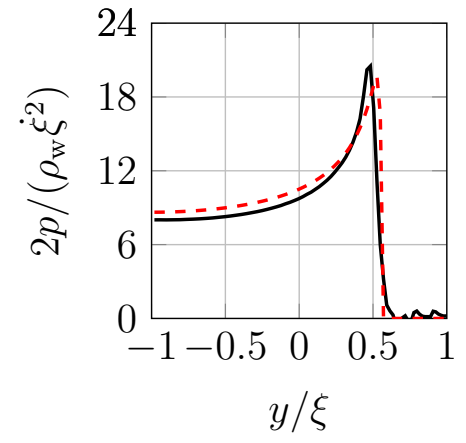

(b) $\beta=20^{\circ}$

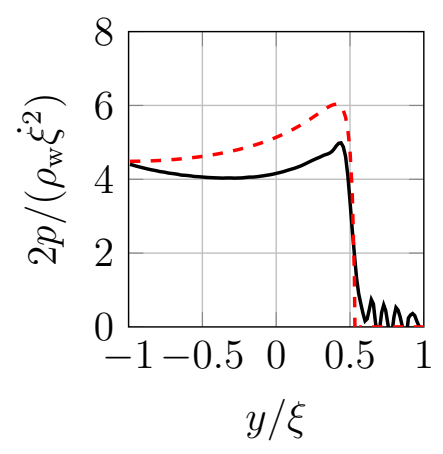

(e) $\beta=35^{\circ}$

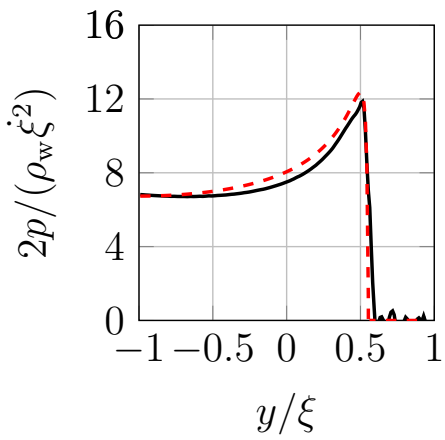

(c) $\beta=25^{\circ}$

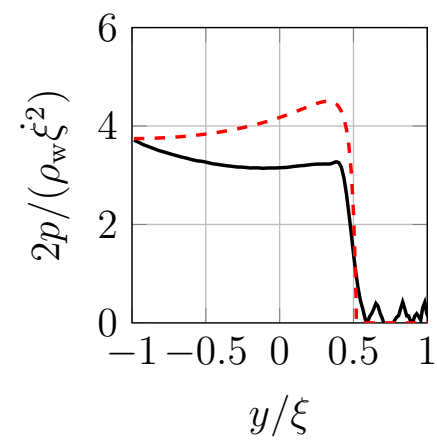

(f) $\beta=40^{\circ}$

Figure A.17: Pressure profile on the wedge for different $\beta$ and a constant penetration velocity. Numerical results are plotted as solid black lines, theoretical findings from Wagner's theory are shown as dashed red lines.

attributed to three-dimensional phenomena which are not addressed in our simulation but are likely to be relevant for a sample with a width equal to half its length as also discussed by Tveitnes et al. (2008). For low deadrise angles, the structural compliance and the difficulty to maintain a constant penetration velocity may be also significant and reduce the force acting on the wedge.

Abrate, S., 2013. Hull slamming. Applied Mechanics Reviews 64, 060803.

Battley, M., Allen, T., 2011. Servo-hydraulic system for controlled velocity water impact of marine sandwich panels. Experimental Mechanics 52, 95-106.

Baur, T., Köngeter, J., 1999. PIV with high temporal resolution for the determination of local pressure reductions from coherent turbulence phenomena, in: International Workshop on PIV'99- Santa Barbara, 3 rd, Santa Barbara, CA, pp. 101-106.

Biscarini, C., Francesco, S.D., Manciola, P., 2010. CFD modelling approach for dam break flow studies. Hydrology and Earth System Sciences 14, 705-718.

Carcaterra, A., Ciappi, E., 2004. Hydrodynamic shock of elastic structures impacting on the water: theory and experiments. Journal of Sound and Vibration 271, $411-439$.

Charca, S., Shafiq, B., Just, F., 2009. Repeated slamming of sandwich composite panels on water. Journal of Sandwich Structures and Materials 11, 409-424.

Colicchio, G., Greco, M., Faltinsen, O.M., 2006. BEM-level set domain decomposition strategy for nonlinear 


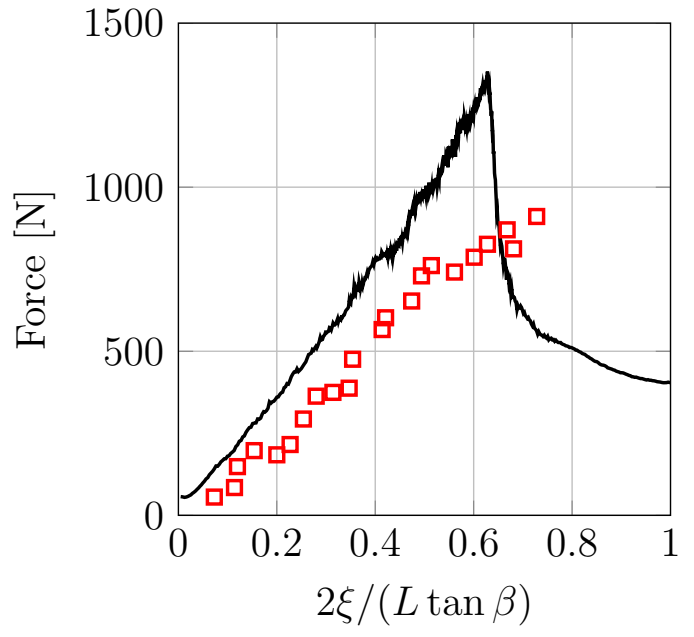

(a) $\beta=15^{\circ}$

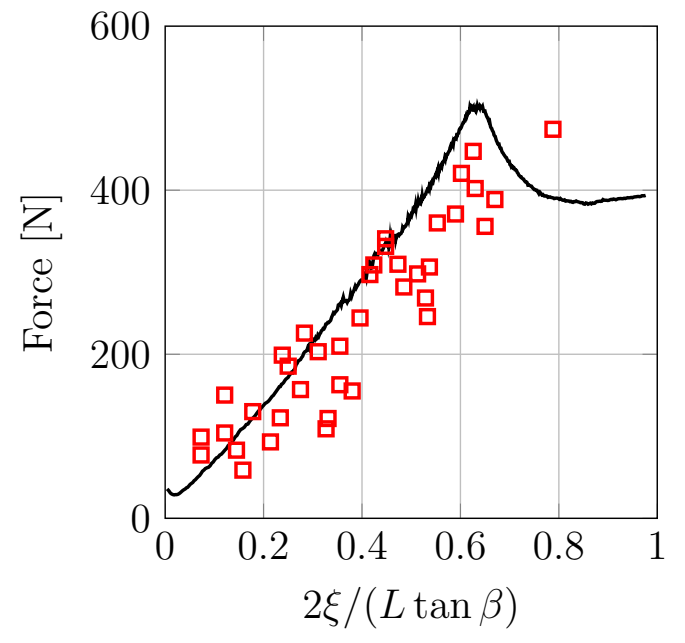

(b) $\beta=30^{\circ}$

Figure A.18: Comparison between CFD results (solid black line) and experimental data by Tveitnes et al. (2008) (red squares).

and fragmented interfacial flows. International Journal for Numerical Methods in Engineering 67, 13851419.

Das, K., Batra, R.C., 2011. Local water slamming impact on sandwich composite hulls. Journal of Fluids and Structures 27, 523-551.

De Rosis, A., Falcucci, G., Porfiri, M., Ubertini, F., Ubertini, S., 2014. Hydroelastic analysis of hull slamming coupling lattice boltzmann and finite element methods. Computers \& Structures 138, 24-35.

Dobrovol'skaya, Z.N., 1969. On some problems of similarity flow of fluid with a free surface. Journal of Fluid Mechanics 36, 805-829.

Faltinsen, O.M., 2000. Hydroelastic slamming. Journal of Marine Science and Technology 5, 49-65.

Faltinsen, O.M., 2005. Hydrodynamics of high-speed marine vehicles. Cambridge University Press.

Ferziger, J.H., Perić, M., 2002. Computational methods for fluid dynamics. volume 3. Springer Berlin.

OpenFOAM Foundation, 2013. OpenFOAM User's guide. Boston, MA, USA.

Garcia, D., 2010. Robust smoothing of gridded data in one and higher dimensions with missing values. Computational Statistics \& Data Analysis 54, 1167-1178.

Gong, K., Liu, H., Wang, B.l., Kai, G., Hua, L., Ben-long, W., 2009. Water entry of a wedge based on SPH model with an imporved boudary treatment. Journal of Hydrodynamics 21, 750-757.

Hirdaris, S.E., Temarel, P., 2009. Hydroelasticity of ships: recent advances and future trends. Proceedings of the Institution of Mechanical Engineers, Part M: Journal of Engineering for the Maritime Environment $223,305-330$.

Hirsch, C., 2007. Numerical computation of internal and external flows: the fundamentals of numerical discretisation. volume 1. Butterworth-Heinemann Oxford.

Hughes, K., Vignjevic, R., Campbell, J., De Vuyst, T., Djordjevic, N., Papagiannis, L., 2013. From aerospace to offshore: bridging the numerical simulation gaps-simulation advancements for fluid structure interaction problems. International Journal of Impact Engineering 61, 48-63.

Issa, R.I., 1985. Solution of the implicitly discretized fluid flow equations by operator-splitting. Journal of Computational Physics 62, 40-65.

Jalalisendi, M., Shams, A., Panciroli, R., Porfiri, M., accepted for publication. Experimental reconstruction of $3 \mathrm{~d}$ hydrodynamic loading in water entry problems through particle image velocimetry. Experiments in 
Fluids .

Jasak, H., 2008. Dynamic mesh handling in OpenFOAM. 47th AIAA Aerospace Sciences Meeting Including the New Horizons Forum and Aerospace Exposition, 5-8 January, Orlando, Florida .

Jasak, H., Tukovic, Z., 2007. Automatic mesh motion for the unstructured finite volume method. Transactions of FAMENA 30, 1-18.

Jasak, H., Weller, H., Gosman, A., 1999. High resolution NVD differencing scheme for arbitrarily unstructured meshes. International Journal for Numerical Methods in Fluids 31, 431-449.

Judge, C., Troesch, A., Perlin, M., 2004. Initial water impact of a wedge at vertical and oblique angles. Journal of Engineering Mathematics 48, 279-303.

Kapsenberg, G.K., 2011. Slamming of ships: where are we now? Philosophical transactions. Series A, Mathematical, physical, and engineering sciences 369, 2892-2919.

Kassiotis, C., 2008. Which strategy to move the mesh in the computational fluid dynamic code OpenFOAM? Technical Report. WiRe, LMT-Cachan, Germany, France, 2008. Http://perso.crans.org/kassiotis/openfoam/movingmesh.pdf.

Khabakhpasheva, T., Korobkin, A.A., 2013. Elastic wedge impact onto a liquid surface: Wagner's solution and approximate models. Journal of Fluids and Structures 36, 32-49.

Korobkin, A.A., 2006. Second-order Wagner theory of wave impact. Journal of Engineering Mathematics $58,121-139$.

Luo, H., Wang, H., Soares, C.G., 2012. Numerical and experimental study of hydrodynamic impact and elastic response of one free-drop wedge with stiffened panels. Ocean Engineering 40, 1-14.

Mei, X., Liu, Y., Yue, D.K., 1999. On the water impact of general two-dimensional sections. Applied Ocean Research 21, 1-15.

Melling, A., 1997. Tracer particles and seeding for particle image velocimetry. Measurement Science and Technology 8, 1406.

Murakami, M., Ichikawa, N., 1989. Flow visualization study of thermal counterflow jet in He II. Cryogenics $29,438-443$.

Nila, A., Vanlanduit, S., Vepa, S., Van Paepegem, W., 2013. A PIV-based method for estimating slamming loads during water entry of rigid bodies. Measurement Science and Technology 24, 045303.

Oger, G., Doring, M., Alessandrini, B., Ferrant, P., 2006. Two-dimensional SPH simulations of wedge water entries. Journal of Computational Physics 213, 803-822.

OpenFoam, 2014. www.openfoam.com.

Panciroli, R., Abrate, S., Minak, G., 2013. Dynamic response of flexible wedges entering the water. Composite Structures 99, 163-171.

Panciroli, R., Abrate, S., Minak, G., Zucchelli, A., 2012. Hydroelasticity in water-entry problems: Comparison between experimental and SPH results. Composite Structures 94, 532-539.

Panciroli, R., Porfiri, M., 2013. Evaluation of the pressure field on a rigid body entering a quiescent fluid through particle image velocimetry. Experiments in fluids 54, 1-13.

Panciroli, R., Shams, A., Porfiri, M., 2015. Experiments on the water entry of curved wedges: high speed imaging and particle image velocimetry. Ocean Engineering 94, 213-222.

Patankar, S., 1980. Numerical Heat Transfer and Fluid Flow. Series in computational methods in mechanics and thermal sciences, Taylor \& Francis.

Peterson, R., Wyman, D., Frank, C., 1997. Drop tests to support water-impact and planing boat dynamics theory. Technical Report CSS/TR-97/25. DTIC Document, Naval surface warfare center panama city fl.

Qin, Z., Batra, R., 2009. Local slamming impact of sandwich composite hulls. International Journal of Solids and Structures 46, 2011-2035.

Raffel, M., Willert, C., Kompenhans, J., 1998. Particle image velocimetry: a practical guide. SpringerVerlag, Berlin, Germany.

Rider, W.J., Kothe, D.B., 1998. Reconstructing volume tracking. Journal of Computational Physics 141, $112-152$.

Rusche, H., 2003. Computational fluid dynamics of dispersed two-phase flows at high phase fractions. Ph.D. thesis. Imperial College London (University of London). 
Scardovelli, R., Zaleski, S., 1999. Direct numerical simulation of free-surface and interfacial flow. Annual Review of Fluid Mechanics 31, 567-603.

Scolan, Y.M., Korobkin, A.A., 2003. Energy distribution from vertical impact of a three-dimensional solid body onto the flat free surface of an ideal fluid. Journal of Fluids and Structures 17, 275-286.

Seng, S., Jensen, J.J., Pedersen, P.T., 2012. Numerical prediction of slamming loads. Proceedings of the Institution of Mechanical Engineers, Part M: Journal of Engineering for the Maritime Environment 226, $120-134$.

Shams, A., Jalalisendi, M., Porfiri, M., accepted for publication. Experiments on the water entry of asymmetric wedges using particle image velocimetry. Physics of Fluids .

Shao, S., 2009. Incompressible SPH simulation of water entry of a free-falling object. International Journal for Numerical Methods in Fluids 59, 91-115.

Stenius, I., Rosén, A., Battley, M., Allen, T., 2013. Experimental hydroelastic characterization of slamming loaded marine panels. Ocean Engineering 74, 1-15.

Sun, H., Faltinsen, O.M., 2006. Water impact of horizontal circular cylinders and cylindrical shells. Applied Ocean Research 28, 299-311.

Tassin, A., Jacques, N., El Malki Alaoui, A., Nême, A., Leblé, B., 2010. Assessment and comparison of several analytical models of water impact. The International Journal of Multiphysics 4, 125-140.

Tassin, A., Piro, D.J., Korobkin, A.A., Maki, K.J., Cooker, M.J., 2013. Two-dimensional water entry and exit of a body whose shape varies in time. Journal of Fluids and Structures 40, 317-336.

Thielicke, W., Stamhuis, E.J., 2014a. PIVlab - time-resolved digital particle image velocimetry tool for MATLAB (version 1.35) .

Thielicke, W., Stamhuis, E.J., 2014b. PIVlab - towards user-friendly, affordable and accurate digital particle image velocimetry in MATLAB. Journal of Open Research Software 2.

Tveitnes, T., Fairlie-Clarke, A., Varyani, K., 2008. An experimental investigation into the constant velocity water entry of wedge-shaped sections. Ocean Engineering 35, $1463-1478$.

Van Oudheusden, B.W., 2013. PIV-based pressure measurement. Measurement Science and Technology 24, 032001.

Von Karman, T., 1929. The impact on seaplane floats, during landing. NACA-TN-321 .

Wagner, H., 1932. Über stoß- und gleitvorgänge an der oberfläche von flüssigkeiten. ZAMM - Zeitschrift für Angewandte Mathematik und Mechanik 12, 193-215.

Willert, C.E., Gharib, M., 1991. Digital particle image velocimetry. Experiments in Fluids 10, 181-193.

Wu, G., Sun, H., He, Y., 2004. Numerical simulation and experimental study of water entry of a wedge in free fall motion. Journal of Fluids and Structures 19, 277-289.

Yettou, E.M., Desrochers, A., Champoux, Y., 2007. A new analytical model for pressure estimation of symmetrical water impact of a rigid wedge at variable velocities. Journal of fluids and structures 23 , $501-522$.

Zarghami, A., Falcucci, G., Jannelli, E., Succi, S., Porfiri, M., Ubertini, S., 2014. Lattice Boltzmann modeling of water entry problems. International Journal of Modern Physics C 25, 1441012.

Zhao, R., Faltinsen, O., 1993. Water entry of two-dimensional bodies. Journal of Fluid Mechanics 246, 593-612.

Zhao, R., Faltinsen, O., Aarsnes, J., 1996. Water entry of arbitrary two-dimensional sections with and without flow separation, in: Proceedings of 21st Symposium on Naval Hydrodynamics, pp. 408-423. 